Document downloaded from:

http://hdl.handle.net/10251/40626

This paper must be cited as:

Bonet Solves, JA.; Albanese, AA.; Ricker, WJ. (2012). Mean ergodic semigroups of operators. Revista de la Real Academia de Ciencias Exactas, Fisicas y Naturales. Serie A. Matematicas. 106(2):299-319. doi:10.1007/s13398-011-0054-2.

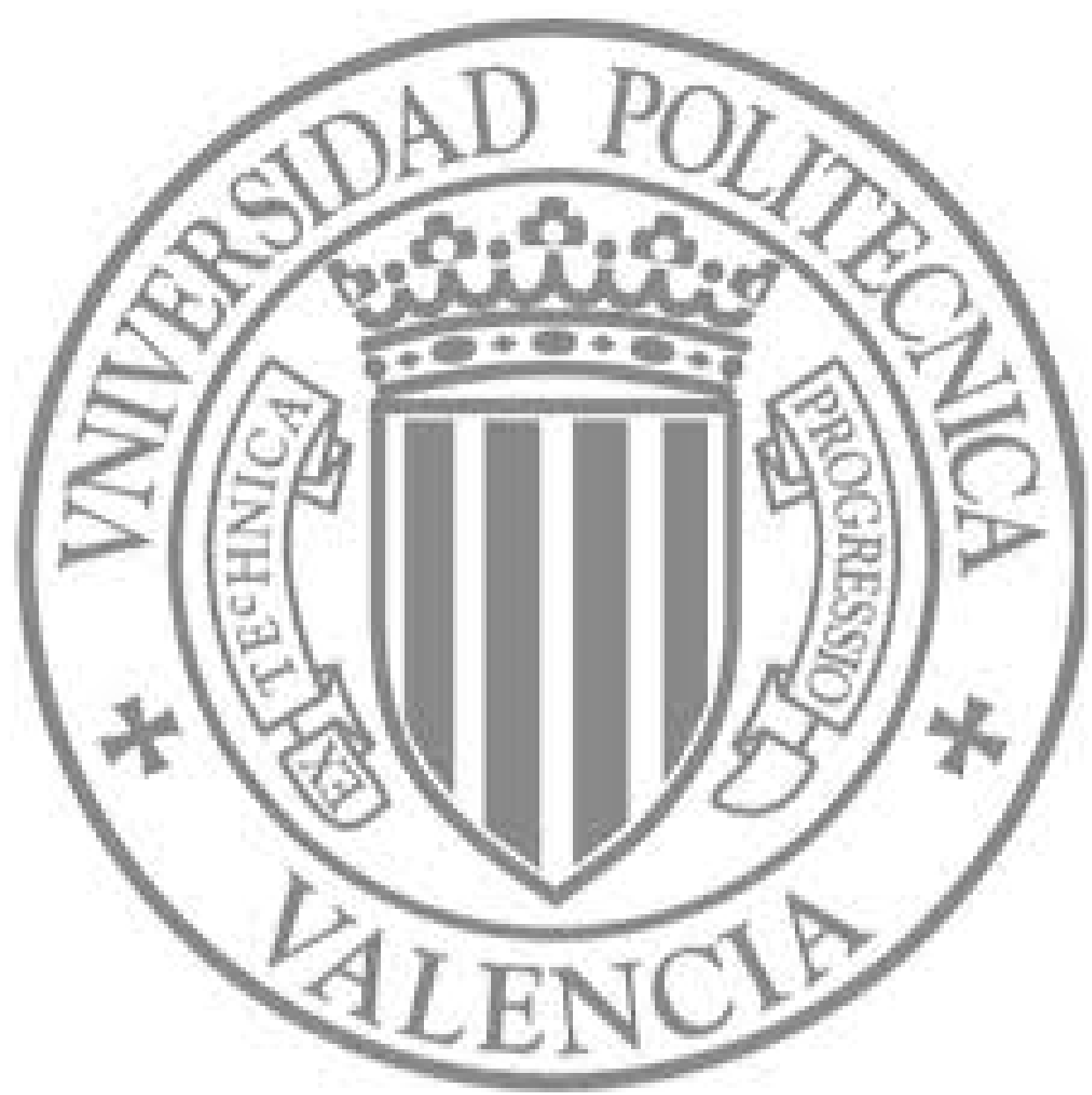

The final publication is available at

http://link.springer.com/article/10.1007/s13398-011-0054-2

Copyright Springer Verlag (Germany) 
Noname manuscript No.

(will be inserted by the editor)

\title{
Mean Ergodic semigroups of operators
}

\author{
Angela A. Albanese • José Bonet ${ }^{*}$. \\ Werner J. Ricker
}

Received: date / Accepted: date

\begin{abstract}
We present criteria for determining mean ergodicity of $C_{0}$-semigroups of linear operators in a sequentially complete, locally convex Hausdorff space $X$. A characterization of reflexivity of certain spaces $X$ with a basis via mean ergodicity of equicontinuous $C_{0}$-semigroups acting in $X$ is also presented. Special results become available in Grothendieck spaces with the Dunford-Pettis property.
\end{abstract}

Keywords $C_{0}$-semigroup $\cdot$ (reflexive) locally convex space $\cdot$ (uniform) mean ergodic

Mathematics Subject Classification (2000) MSC 46A04 • 47A35 . 47D03 - 46A11

\footnotetext{
Project Prometeo/2008/101.

Angela A. Albanese

Dipartimento di Matematica "E.De Giorgi"

Università del Salento- C.P.193

I-73100 Lecce, Italy

E-mail: angela.albanese@unisalento.it

José Bonet

Instituto Universitario de Matemática Pura y Aplicada IUMPA

Edificio IDI5 (8E), Cubo F, Cuarta Planta

Universitat Politècnica de València

E-46071 Valencia, Spain

E-mail: jbonet@mat.upv.es

Werner J. Ricker

Math.-Geogr. Fakultät

Katholische Universität Eichstätt-Ingolstadt

D-85072 Eichstätt, Germany

E-mail: werner.ricker@ku-eichstaett.de
}

* Research partially supported by MICINN and FEDER Project MTM2010-15200 and GV 


\section{Introduction}

For a continuous linear operator $T$ in a Banach space $X$, its Cesàro means are defined by

$$
T_{[n]}:=\frac{1}{n} \sum_{m=0}^{n-1} T^{m}, \quad n \in \mathbb{N} .
$$

If $\left\{T_{[n]}\right\}_{n=1}^{\infty}$ is convergent in the strong operator topology, then $T$ is called mean ergodic. The interest in such operators has its origins in statistical mechanics and probability theory. In such settings, one also considers continuous processes $\phi_{t}$, with $t$ specifying time, which in many situations satisfy $\phi_{t}\left(\phi_{s}(u)\right)=\phi_{t+s}(u)$ for all points $u$ in a phase space and all times $s, t$. The abstract setting then consists of a semigroup of continuous linear operators $(T(t))_{t \geq 0}$ acting in $X$ (i.e., $T(s+t)=T(s) T(t)$ for all $s, t \geq 0$ ) and one investigates the long term behaviour of $(T(t))_{t>0}$ via its Cesàro averages $C(r):=r^{-1} \int_{0}^{r} T(t) d t$ for $r>0$. If this limit exists in the strong operator topology, then $(T(t))_{t \geq 0}$ is called mean ergodic. Fix $t_{0}>0$. Given $n \in \mathbb{N}$ it turns out that $C\left(n t_{0}\right)=T\left(t_{0}\right)_{[n]} C\left(t_{0}\right)$ and one sees the connection between the Cesàro averages $\left\{C\left(n t_{0}\right)\right\}_{n=1}^{\infty}$ of the semigroup $(T(t))_{t \geq 0}$ and the discrete Cesàro means $\left\{T\left(t_{0}\right)_{[n]}\right\}_{n=1}^{\infty}$ of the individual operator $T\left(t_{0}\right)$. So, there is a need to simultaneously investigate individual mean ergodic operators and mean ergodic systems of operators (i.e., semigroups). This has happened ever since the origins of the subject (i.e., the 1930's) and both theories are adequately addressed in the monographs, [8, Ch.VIII], [14, Ch.XVIII], [21], for example.

Much of modern analysis occurs in locally convex Hausdorff spaces (briefly, lcHs) which are non-normable. So, there is an interest in extending the Banach space results for mean ergodicity to this setting. For individual operators (especially Banach space results related to the theory of bases, [13]) this has been carried out in recent years, [1], [3], [4], [6], [7], [27], [28]. For certain aspects of the theory of mean ergodic semigroups of operators in the non-normable setting we refer to [9], [21, Ch.2], [31, Ch.III, §7], and the references therein. The aim of this paper is to develop this topic further, also in some rather different directions.

The natural framework is a sequentially complete lcHs $X$ and a $C_{0}$-semigroup of continuous linear operators $(T(t))_{t \geq 0}$ acting in $X$. An adequate theory is available if $(T(t))_{t \geq 0}$ is equicontinuous, [33, Ch.IX]. However, this is too restrictive as there exist examples of non-equicontinuous $C_{0}$-semigroups; see $§ 2$. For $X$ a Fréchet space, the theory of certain types of non-equicontinuous $C_{0^{-}}$ semigroups (called locally equicontinuous) is developed in [25]. But, even the Fréchet space setting is somewhat restrictive; this condition on $X$ is relaxed in [18]. To define the Cesàro averages $\{C(r)\}_{r \geq 0}$ and establish some of their properties requires aspects of vector-valued Riemann integration; the relevant information is given in an Appendix.

In Section 3 results concerning mean ergodicity of certain classes of $C_{0^{-}}$ semigroups of operators $(T(t))_{t>0}$ are established. We present sufficient conditions for various classes of $C_{0}$-semigroups to be mean ergodic (eg. Proposition 
3, Corollary 2 and Theorem 4). A basic result needed in this regard is the fundamental mean ergodic theorem of W.F. Eberlein, [9]. In Theorem 5 we characterize the reflexivity of a complete, barrelled lcHs $X$ with a Schauder basis via the condition that every equicontinuous $C_{0}$-semigroup of operators in $X$ should be mean ergodic; for Banach spaces with a basis, see [26].

Section 4 concerns $C_{0}$-semigroups $(T(t))_{t \geq 0}$ in Grothendieck lcHs' with the Dunford-Pettis property (briefly, GDP) and their mean ergodicity. Certain classes of such $C_{0}$-semigroups are automatically uniformly continuous (cf. Theorem 7). Criteria are given which ensure that the dual semigroup $\left(T(s)^{\prime}\right)_{s \geq 0}$ is strongly continuous in $X_{\beta}^{\prime}$ (the strong dual of $X$ ). Mean ergodicity of $(T(t))_{t \geq 0}$ in Grothendieck spaces is equivalent to the subspace span $\left\{x^{\prime}-T(s)^{\prime} x^{\prime}: x^{\prime} \in X^{\prime}, s \geq 0\right\}$ of $X^{\prime}$ having the same closure for the weak-star topology as in $X_{\beta}^{\prime}$; see Theorem 9 . Consequently, if $(T(t))_{t \geq 0}$ is a mean ergodic, strongly continuous $C_{0}$-semigroup in a Grothendieck space and $\lim _{t \rightarrow \infty} T(t) / t=0$ (for the topology of uniform convergence on bounded sets of $X)$, then also $\left(T(s)^{\prime}\right)_{s>0}$ is mean ergodic in $X_{\beta}^{\prime}$. For GDP-Banach spaces, some of these results are due to H.P.Lotz, [22], [23].

\section{Preliminaries.}

Let $X$ be a locally convex Hausdorff space (briefly, lcHs) and $\Gamma_{X}$ a system of continuous seminorms determining the topology of $X$. The strong operator topology $\tau_{s}$ in the space $\mathcal{L}(X)$ of all continuous linear operators from $X$ to itself (from $X$ to another lcHs $Y$ we write $\mathcal{L}(X, Y)$ ) is determined by the seminorms $q_{x}(S):=q(S x)$, for $S \in \mathcal{L}(X)$, with $x \in X$ and $q \in \Gamma_{X}$, in which case we write $\mathcal{L}_{s}(X)$. Denote by $\mathcal{B}(X)$ the collection of all bounded subsets of $X$. The topology $\tau_{b}$ of uniform convergence on bounded sets is defined in $\mathcal{L}(X)$ via the seminorms $q_{B}(S):=\sup _{x \in B} q(S x)$, for $S \in \mathcal{L}(X)$, with $B \in \mathcal{B}(X), q \in \Gamma_{X}$; in this case we write $\mathcal{L}_{b}(X)$. The identity operator on $X$ is denoted by $I$.

By $X_{\sigma}$ we denote $X$ with its weak topology $\sigma\left(X, X^{\prime}\right)$, where $X^{\prime}$ is the topological dual space of $X$. The strong topology in $X$ (resp. $X^{\prime}$ ) is denoted by $\beta\left(X, X^{\prime}\right)$ (resp. $\left.\beta\left(X^{\prime}, X\right)\right)$ and we write $X_{\beta}$ (resp. $\left.X_{\beta}^{\prime}\right)$; see $[19, \S 21.2]$ for the definition. Then $\beta\left(X^{\prime}, X\right)$ is generated by the seminorms $p^{B}\left(x^{\prime}\right):=$ $\sup _{x \in B}\left|\left\langle x, x^{\prime}\right\rangle\right|$, for $x^{\prime} \in X^{\prime}$, with $B \in \mathcal{B}(X)$. The strong dual $\left(X_{\beta}^{\prime}\right)_{\beta}^{\prime}$ of $X_{\beta}^{\prime}$ is denoted by $X^{\prime \prime}$. By $X_{\sigma^{*}}^{\prime}$ we denote $X^{\prime}$ with its weak-star topology $\sigma\left(X^{\prime}, X\right)$. Given $T \in \mathcal{L}(X)$, its dual operator $T^{\prime}: X^{\prime} \rightarrow X^{\prime}$ is defined by $\left\langle x, T^{\prime} x^{\prime}\right\rangle=$ $\left\langle T x, x^{\prime}\right\rangle$ for $x \in X, x^{\prime} \in X^{\prime}$. Then $T^{\prime} \in \mathcal{L}\left(X_{\sigma}^{\prime}\right)$ and $T^{\prime} \in \mathcal{L}\left(X_{\beta}^{\prime}\right)$, [20, p.134].

Definition 1 Let $X$ be a lcHs and $(T(t))_{t \geq 0} \subseteq \mathcal{L}(X)$ be a 1-parameter family of operators. The map $t \mapsto T(t)$, for $t \in[0, \infty)$, is denoted by $T:[0, \infty) \rightarrow$ $\mathcal{L}(X)$.

We say that $(T(t))_{t \geq 0}$ is a semigroup if it satisfies

(i) $T(s) T(t)=T(s+t)$ for $s, t \geq 0$, with $T(0)=I$.

A semigroup $(T(t))_{t \geq 0}$ is locally equicontinuous if, for fixed $K>0$, the set $\{T(t): 0 \leq t \leq K\}$ is equicontinuous, i.e., given $p \in \Gamma_{X}$ there exist $q \in \Gamma_{X}$ 
and $M>0$ with

$$
p(T(t) x) \leq M q(x), \quad x \in X, t \in[0, K] .
$$

A semigroup $(T(t))_{t \geq 0}$ is said to be a $C_{0}$-semigroup if it satisfies

(ii) $\lim _{t \rightarrow 0^{+}} T(t)=I$ in $\mathcal{L}_{s}(X)$.

If the $C_{0}$-semigroup $(T(t))_{t \geq 0}$ satisfies the additional condition that

(iii) $\lim _{t \rightarrow t_{0}} T(t)=T\left(t_{0}\right)$ in $\mathcal{L}_{s}(X)$, for each $t_{0}>0$,

then it is called a strongly continuous $C_{0}$-semigroup.

A semigroup $(T(t))_{t \geq 0}$ is said to be exponentially equicontinuous if there exists $a \geq 0$ such that $\left(e^{-a t} T(t)\right)_{t \geq 0} \subseteq \mathcal{L}(X)$ is equicontinuous, i.e.,

$$
\forall p \in \Gamma_{X} \exists q \in \Gamma_{X}, M_{p}>0 \text { with } p(T(t) x) \leq M_{p} e^{a t} q(x) \forall t \geq 0, x \in X .
$$

If $a=0$, then we simply say equicontinuous. Finally, a semigroup $(T(t))_{t \geq 0}$ is called uniformly continuous if $T:[0, \infty) \rightarrow \mathcal{L}_{b}(X)$ is continuous, i.e.,

(iv) $\lim _{t \rightarrow t_{0}} T(t)=T\left(t_{0}\right)$ in $\mathcal{L}_{b}(X)$, for each $t_{0}>0$ (with $t \rightarrow 0^{+}$if $t_{0}=0$ ).

Remark 1 (i) If $X$ is barrelled, then every strongly continuous $C_{0}$-semigroup is locally equicontinuous, [18, Proposition 1.1].

(ii) Every exponentially equicontinuous semigroup is locally equicontinuous. A strongly continuous $C_{0}$-semigroup in a Banach space is always exponentially equicontinuous, [8, p.619]. For Fréchet spaces this need not be so. Indeed, in the sequence space $\omega=\mathbb{C}^{\mathbb{N}}$ (topology of coordinate convergence), $T(t) x:=\left(e^{n t} x_{n}\right)_{n=1}^{\infty}$, for $t \geq 0$ and $x=\left(x_{n}\right)_{n=1}^{\infty} \in \omega$, is a strongly continuous $C_{0}$-semigroup which is not exponentially equicontinuous. Since $\omega$ is barrelled, by (i) we see $(T(t))_{t \geq 0}$ is locally equicontinuous. As $\omega$ is a Montel space, $(T(t))_{t \geq 0}$ is also uniformly continuous. For an example in a Fréchet space of smooth functions, see [25, pp.162-163].

(iii) A locally equicontinuous $C_{0}$-semigroup $(T(t))_{t \geq 0}$ is strongly continuous. Indeed, via Definition 1(i) we have $T\left(t_{0}+h\right)-T\left(t_{0}\right)=T\left(t_{0}\right)(T(h)-I)$ for $h \geq 0$ and fixed $t_{0}>0$. It follows from continuity of $T\left(t_{0}\right)$ and Definition 1(ii) that $T:[0, \infty) \rightarrow \mathcal{L}_{s}(X)$ is right continuous at points of $[0, \infty)$. Fix $t_{0}>0$. Given $p \in \Gamma_{X}$, let $K:=t_{0}$ and choose $q \in \Gamma_{X}$ and $M>0$ so that (2) holds. Then

$$
p\left(T\left(t_{0}+h\right) x-T\left(t_{0}\right) x\right)=p\left(T\left(t_{0}+h\right)(x-T(-h) x)\right) \leq M q(x-T(-h) x),
$$

for $x \in X$ and $h \in\left[-t_{0} / 2,0\right]$. In combination with Definition 1(ii) this implies left continuity of $T:[0, \infty) \rightarrow \mathcal{L}_{s}(X)$ at $t_{0}$.

(iv) Let $(T(t))_{t \geq 0} \subseteq \mathcal{L}(X)$ be an equicontinuous semigroup. For $p \in \Gamma_{X}$ define $\tilde{p}(x):=\sup _{t \geq 0} p(T(t) x)$, for $x \in X$. Then $\tilde{p}$ is well-defined, is a seminorm and

$$
p(x) \leq \tilde{p}(x) \leq M_{p} q(x) \leq M_{p} \tilde{q}(x), \quad x \in X,
$$

with $M_{p}>0, q \in \Gamma_{X}$ chosen via (3) (for $a=0$ ). So, $\tilde{\Gamma}_{X}:=\left\{\tilde{p}: p \in \Gamma_{X}\right\}$ also generates the given lc-topology of $X$. Moreover, for $\tilde{p} \in \tilde{\Gamma}_{X}$, we have

$$
\tilde{p}(T(t) x)=\sup _{s \geq 0} p(T(t) T(s) x)=\sup _{s \geq 0} p(T(t+s) x) \leq \tilde{p}(x), \quad x \in X, t \geq 0 .
$$


A similar result to Proposition 1.6 of [17] (in Banach spaces see [14, Corollary, p.306, Theorem 10.6.5]) holds for semigroups of operators in lcHs'.

Proposition 1 Let $X$ be a quasicomplete lcHs and $(T(t))_{t \geq 0} \subseteq \mathcal{L}(X)$ be a locally equicontinuous semigroup. Then $(T(t))_{t \geq 0}$ is a $C_{0}-$ semigroup if and only if $t \mapsto\left\langle T(t) x, x^{\prime}\right\rangle$ is continuous on $[0, \infty)$ for each $x \in X, x^{\prime} \in X^{\prime}$.

Proof If $(T(t))_{t \geq 0}$ is a $C_{0}$-semigroup, then $T:[0, \infty) \rightarrow \mathcal{L}_{s}(X)$ is continuous (cf. Remark $1(\mathrm{iii})$ ) and so $\left\langle T(\cdot) x, x^{\prime}\right\rangle$ is continuous on $[0, \infty)$ for each $x \in X$, $x^{\prime} \in X^{\prime}$.

Assume then that $\left\langle T(\cdot) x, x^{\prime}\right\rangle$ is continuous for each $x \in X, x^{\prime} \in X^{\prime}$. Fix $x \in$ $X$. For $r>0$, the function $\varphi_{r, x}(s):=T(s) x, s \in[0, r]$, is $\sigma\left(X, X^{\prime}\right)$-continuous. So, $\varphi_{r, x}([0, r])$ is compact in $X_{\sigma}$. Since $X$ is also quasicomplete for its Mackey topology, [19, 18.4.4, p.210], Krein's theorem implies the closed convex hull $H_{r, x}$ of $\varphi_{r, x}([0, r])$ is compact in $X_{\sigma},\left[19,24.5,4^{\prime}, p .325\right]$. It follows from [29, Theorem 3.27] applied in $X_{\sigma}$ that the weak integral $I(r, x):=\frac{1}{r} \int_{0}^{r} \varphi_{r, x}(s) d s \in$ $H_{r, x}$ exists in the sense that $\left\langle I(r, x), x^{\prime}\right\rangle=\frac{1}{r} \int_{0}^{r}\left\langle T(s) x, x^{\prime}\right\rangle d s$, for $x^{\prime} \in X^{\prime}$. Since $s \mapsto\left\langle T(s) x, x^{\prime}\right\rangle$ is continuous on $[0, r]$, the theory of scalar integration yields

$$
\lim _{r \rightarrow 0^{+}} \frac{1}{r} \int_{0}^{r}\left\langle T(s) x, x^{\prime}\right\rangle d s=\left\langle T(0) x, x^{\prime}\right\rangle=\left\langle x, x^{\prime}\right\rangle, \quad x^{\prime} \in X^{\prime} .
$$

That is, $\sigma\left(X, X^{\prime}\right)-\lim _{r \rightarrow 0^{+}} I(r, x)=x$, for each $x \in X$. In particular, the subspace $H:=\operatorname{span}\{I(r, x): x \in X, r>0\}$ is dense in $X$ (being dense in $\left.X_{\sigma}\right)$.

To complete the proof that $(T(t))_{t \geq 0}$ is a $C_{0}$-semigroup, it suffices to show $\lim _{t \rightarrow 0^{+}} T(t) y=y$, for $y \in H$, as $H$ is dense with $\{T(t): t \in[0,1]\} \subseteq \mathcal{L}(X)$ equicontinuous and so the existence of $\lim _{t \rightarrow 0^{+}} T(t) x=x$ for an arbitrary $x \in X$ follows from [20,39.4.1, p.138]. To this effect, fix $r>0, x \in X$ and set $y:=I(r, x)$. For $s \in(0,1]$ we have $y-T(s) y=I(r, x)-T(s) I(r, x)$. Applying $x^{\prime} \in X^{\prime}$ and arguing as in the proof of Lemma 1(v) below, with the weak integral $I(r, x)$ replacing the Riemann integral $\frac{1}{r} \int_{0}^{r} T(s) x d s$, yields $y-T(s) y=$ $\frac{1}{r}(I-T(r)) s I(s, x)$. But, $s I(s, x) \in s H_{s, x} \subseteq s H_{1, x}$ with $H_{1, x}$ compact in $X_{\sigma}$ and hence, bounded. As $T(r) \in \mathcal{L}\left(X_{\sigma}\right)$, the set $T(r) H_{1, x}$ is also compact in $X_{\sigma}$. Moreover, $y-T(s) y \in \frac{s}{r}\left(H_{1, x}+T(r) H_{1, x}\right)$. Since $\frac{1}{r}\left(H_{1, x}+T(r) H_{1, x}\right) \in \mathcal{B}(X)$ and is independent of $s$, we conclude that $\lim _{s \rightarrow 0^{+}}(y-T(s) y)=0$.

If $X$ is a sequentially complete $\mathrm{lcHs}$ and $(T(t))_{t \geq 0}$ is a locally equicontinuous $C_{0}$-semigroup on $X$, then the linear operator $A$ defined by

$$
A x:=\lim _{t \rightarrow 0^{+}} \frac{T(t) x-x}{t},
$$

for $x \in D(A):=\left\{x \in X: \lim _{t \rightarrow 0^{+}} \frac{T(t) x-x}{t}\right.$ exists in $\left.X\right\}$, is closed with $\overline{D(A)}=X,[18$, Propositions $1.3 \& 1.4]$. The operator $(A, D(A))$ is called the infinitesimal generator of $(T(t))_{t \geq 0}$. Moreover, $A$ and $(T(t))_{t \geq 0}$ commute, $[18$, Proposition 1.2(1)], i.e., for each $t \geq 0$ we have $\{T(t) x: x \in D(A)\} \subseteq D(A)$ 
and $A T(t) x=T(t) A x$ for all $x \in D(A)$. Also known, [18, Proposition 1.2(2)], is that

$$
T(t) x-x=\int_{0}^{t} T(s) A x d s=\int_{0}^{t} A T(s) x d s, \quad x \in D(A) .
$$

For each $x \in X$ and $t>0$, the integrals occuring in (6) are Riemann integrals of an $X$-valued, continuous function on $[0, t]$; see Section 5 . The closedness of $A$ ensures that $\operatorname{Ker} A:=\{x \in D(A): A x=0\}$ is a closed subspace of $X$.

\section{Mean ergodic theorems for $C_{0}$-semigroups in lcHs}

Let $X$ be a lcHs and $T \subseteq \mathcal{L}(X)$ be a semigroup under composition. Following Eberlein, [9, Definitiom 2.1], [21, Chapter 2, §2.1, p.75], we recall that $T$ admits a $T$-ergodic net $\left\{A_{\lambda}: \lambda \in \Lambda\right\}$ if $\Lambda$ is a directed set and

(E1) $A_{\lambda} \in \mathcal{L}(X)$ for all $\lambda \in \Lambda$,

(E2) $A_{\lambda} x \in \overline{\operatorname{co}\{S x: S \in T\}}$, for all $x \in X, \lambda \in \Lambda$,

(E3) $\left\{A_{\lambda}: \lambda \in \Lambda\right\}$ is equicontinuous,

(E4l) for every $x \in X$ and $S \in T$

$$
\lim _{\lambda}\left(S A_{\lambda} x-A_{\lambda} x\right)=0,
$$

(E4r) for every $x \in X$ and $S \in T$

$$
\lim _{\lambda}\left(A_{\lambda} S x-A_{\lambda} x\right)=0 .
$$

Define a subspace of $X,[21, \mathrm{p} .77]$, necessarily closed in $X$, by

$$
\operatorname{Fix}(T):=\{x \in X: S x=x, \forall S \in T\}=\cap_{S \in T} \operatorname{Ker}(I-S) .
$$

We recall Eberlein's Theorem, [9, Theorem 3.1], [21, Chapter 2, Theorem 1.5, p.76];

Theorem 2 If $T=(T(t))_{t \geq 0} \subseteq \mathcal{L}(X)$ is a 1-parameter semigroup on a lcHs $X$ admitting a T-ergodic net $\left\{A_{\lambda}: \lambda \in \Lambda\right\}$ then, for every $x, y \in X$, the following assertions are equivalent.

(i) $y \in \operatorname{Fix}(T(\cdot))$ and $y \in \overline{\operatorname{co}\{T(t) x: 0 \leq t<\infty\}}$.

(ii) $y=\lim _{\lambda} A_{\lambda} x$.

(iii) $y=\sigma\left(X, X^{\prime}\right)-\lim _{\lambda} A_{\lambda} x$.

(iv) $y$ is a $\sigma\left(X, X^{\prime}\right)$ cluster point of $\left\{A_{\lambda} x: \lambda \in \Lambda\right\}$.

Remark 2 The implications (ii) $\Rightarrow($ iii) $\Rightarrow$ (iv) of Theorem 2 (as proved in [9], [21]) are clear. For the proof of (iv) $\Rightarrow(\mathrm{i})$ one only needs (E1), (E2) and (E4l). Furthermore, the equicontinuity of $\left\{A_{\lambda}: \lambda \in \Lambda\right\}$ is unnecessary for (iv) $\Rightarrow(\mathrm{i})$. Finally, the proof of (i) $\Rightarrow$ (ii) does not require (E4l). 
In the rest of this Section we suppose that $T=(T(t))_{t \geq 0}$ is a locally equicontinuous $C_{0}$-semigroup on a sequentially complete lcHs $X$. Hence, $T$ is always strongly continuous (cf. Remark $1(\mathrm{iii})$ ). The linear operators $C(0):=I$ and $C(r)$, for $r>0$, given by

$$
x \mapsto C(r) x:=\frac{1}{r} \int_{0}^{r} T(t) x d t, \quad x \in X,
$$

are called the Cesàro means of $(T(t))_{t \geq 0}$. For $x \in X$ and $r>0$, the integral in (7) is the Riemann integral of the continuous $X$-valued function $t \mapsto T(t) x$, for $t \in[0, r]$, relative to the lc-topology of $X$; see Section 5 .

The Cesàro means $\{C(r)\}_{r \geq 0}$ belong to $\mathcal{L}(X)$. Indeed, by local equicontinuity, for fixed $r>0$ and $p \in \bar{\Gamma}_{X}$ there exist $q \in \Gamma_{X}$ and $M>0$ (depending on $p, r)$ with

$$
p(T(t) x) \leq M q(x), \quad x \in X, 0 \leq t \leq r,
$$

and hence, by Proposition 11(vii), we have that

$$
p(C(r) x) \leq \frac{1}{r} \int_{0}^{r} p(T(t) x) d t \leq M q(x), \quad x \in X .
$$

This implies $C(r) \in \mathcal{L}(X)$ for $r>0$. The definition of the Riemann integral $\int_{0}^{r} T(t) x d t$ as a limit of Riemann sums, together with continuity of $T(t), C(r) \in \mathcal{L}(X)$, implies $T(t) C(r)=C(r) T(t)$ for $r, t \geq 0$. In case $X$ is barrelled, the Cesàro means are well defined in $\mathcal{L}(X)$ whenever the $C_{0^{-}}$ semigroup $(T(t))_{t \geq 0}$ is strongly continuous (via Remark 1(i)). Moreover, if $(T(t))_{t \geq 0}$ is exponentially equicontinuous, then (3) and (9) imply (in the notation of (3)) that $p\left(e^{-a r} C(r) x\right) \leq M_{p} q(x)$, for $x \in X, r>0$. Whenever $(T(t))_{t \geq 0}$ is equicontinuous, the seminorm $q$ in (8) and (9) can be chosen independent of $r \geq 0$ and so $\{C(r)\}_{r \geq 0}$ is also equicontinuous.

According to the following result the Cesàro means $\{C(r)\}_{r \geq 0}$, which commute with $(T(t))_{t \geq 0}$, satisfy some of the properties of a $T$-ergodic net.

Lemma 1 Let $X$ be a sequentially complete lcHs and $(T(t))_{t \geq 0}$ be a locally equicontinuous $C_{0}$-semigroup on $X$. Then the following assertions are valid.

(i) $C(r) x \in \overline{\operatorname{co}\{T(t) x: 0 \leq t \leq r\}}$, for all $x \in X, r \geq 0$.

(ii) $x-C(r) x \in \overline{\operatorname{span}\{u-T(t) u: u \in X, t \geq 0\}}$, for all $x \in X, r \geq 0$.

(iii) $\lim _{r \rightarrow 0^{+}} C(r)=I=C(0)$ in $\mathcal{L}_{s}(X)$.

(iv) The $X$-valued function $r \mapsto r C(r) x$ is continuous in $[0, \infty)$ for every $x \in X$.

(v) The following identities hold:

$$
(I-T(t)) C(r)=C(r)(I-T(t))=\frac{1}{r}(I-T(r)) \int_{0}^{t} T(s) d s, \quad r, t>0 .
$$

(vi) The function $r \mapsto C(r)$ is continuous from $[0, \infty)$ into $\mathcal{L}_{s}(X)$. 
Proof (i) Follows from Proposition 11(iv).

(ii) This follows from $x-C(r) x=\frac{1}{r} \int_{0}^{r}(x-T(s) x) d s$, for $x \in X$ and $r>0$, and Proposition 11(iv).

(iii) Fix $x \in X$. Let $p \in \Gamma_{X}$ and $\varepsilon>0$. As $(T(t))_{t>0}$ is a $C_{0}$-semigroup, there is $R>0$ such that $p(x-T(s) x)<\varepsilon$, for $s \in[0, R]$. Since $x-C(r) x=$ $\frac{1}{r} \int_{0}^{r}(x-T(s) x) d s$ for $r>0$, it then follows from Proposition 11(vii) that

$$
p(x-C(r) x) \leq \frac{1}{r} \int_{0}^{r} p(x-T(s) x) d s<\varepsilon, \quad r \in(0, R] .
$$

Since $p$ and $\varepsilon$ are arbitrary we deduce that $\lim _{r \rightarrow 0^{+}} C(r) x=x$.

(iv) Fix $x \in X$ and $K>0$. Suppose that $s, r \in(0, K)$, say $0<s<r$. Proposition 11(ii) implies that $r C(r) x-s C(s) x=\int_{s}^{r} T(t) x d t$. Given $p \in$ $\Gamma_{X}$ it then follows that $p(r C(r) x-s C(s) x)=p\left(\int_{s}^{r} T(s) T(t-s) x d t\right)$. Apply Proposition 11(vi) and then Proposition 11(iii) yields $p(r C(r) x-s C(s) x)=$ $p\left(T(s) \int_{s}^{r} T(t-s) x d t\right)=p\left(T(s) \int_{0}^{r-s} T(u) x d u\right)$. By local equicontinuity of $(T(t))_{t \geq 0}$ there exist $q \in \Gamma_{X}$ and $M>0$ such that (2) holds. So, by the previous identity, $p(r C(r) x-s C(s) x) \leq M q\left(\int_{0}^{r-s} T(u) x d u\right)$. Then Proposition 11(vii) gives

$$
p(r C(r) x-s C(s) x) \leq(r-s) M \sup \{q(T(u) x): u \in[0, K]\}
$$

with $\sup \{q(T(u) x): u \in[0, K]\}<\infty$ by compactness of $[0, K]$ and continuity of $u \mapsto T(u) x$. It is clear from (11) that $r \mapsto r C(r) x$ is continuous in $(0, \infty)$. Continuity of $r \mapsto r C(r) x$ at $r=0$ follows from part (iii).

(v) From the definition, for $x \in X$ fixed, we have

$$
(I-T(t)) C(r) x=\frac{1}{r}\left(\int_{0}^{r} T(u) x d u-T(t) \int_{0}^{r} T(v) x d v\right) .
$$

So, to verify (10) it suffices to show that $\int_{0}^{r} T(u) x d u-T(t) \int_{0}^{r} T(v) x d v=$ $(I-T(r)) \int_{0}^{t} T(s) x d s$ or, by rearranging, that $\int_{0}^{t} T(s) x d s-\int_{0}^{r} T(u) x d u=$ $T(r) \int_{0}^{t} T(s) x d s T(t) \int_{0}^{r} T(v) x d v$. In view of Proposition 11(ii), with $r \leq t$ say, this is equivalent to showing the identity $\int_{r}^{t} T(s) x d s=T(r) \int_{0}^{t} T(s) x d s-$ $T(t) \int_{0}^{r} T(v) x d v$. By applying Proposition 11(vi) to the right side of this identity and using the semigroup property, we only need to verify

$$
\int_{r}^{t} T(s) x d s=\int_{0}^{t} T(r+s) x d s-\int_{0}^{r} T(t+v) x d v .
$$

But, by a change of variables, the right side of (12) is precisely $\int_{r}^{r+t} T(w) x d w-$ $\int_{t}^{r+t} T(w) x d w$ (cf. Proposition 11(iii)) which, in view of Proposition 11(ii), is precisely the left side of (12), as required.

(vi) This is a consequence of parts (iii) and (iv). 
Remark 3 As noted prior to Lemma 1, the maps $\{C(r)\}_{r \geq 0}$ satisfy (E1). Moreover, Lemma 1 ensures that (E2) is also satisfied by $\{\bar{C}(r)\}_{r \geq 0}$ and implies the equivalence of

(i) $\{C(r)\}_{r \geq 0}$ satifies (E4l),

(ii) $\{C(r)\}_{r \geq 0}$ satisfies (E4r),

(iii) $\tau_{s}-\lim _{r \rightarrow \infty} \frac{1}{r} T(r) \int_{0}^{t} T(s) d s=0$ for all $t>0$.

A locally equicontinuous $C_{0}$-semigroup $(T(t))_{t \geq 0} \subseteq \mathcal{L}(X)$ is mean ergodic if the net $\{C(r)\}_{r \geq 0}$ converges to some operator in $\mathcal{L}_{s}(X)$ as $r \rightarrow \infty$. If $\{C(r)\}_{r \geq 0}$ converges in $\mathcal{L}_{b}(X)$ as $r \rightarrow \infty$, then $(T(t))_{t \geq 0}$ is said to be uniformly mean ergodic.

Also, recall that a subset $A \subseteq X$ is relatively sequentially compact (resp. relatively sequentially $\sigma\left(X, X^{\prime}\right)$-compact) if every sequence in $A$ has a convergent subsequence in $X$ (resp. in $X_{\sigma}$ ).

By invoking Theorem 2, we now collect various results concerning mean ergodicity of semigroups.

Remark 4 (i) Assume that the locally equicontinuous $C_{0}$-semigroup $T=(T(t))_{t \geq 0}$ satisfies the requirement

$$
\lim _{t \rightarrow \infty} \frac{1}{t} T(t)=0 \text { in } \mathcal{L}_{s}(X) .
$$

Then condition (iii) of Remark 3 is satisfied. Accordingly, if for $x \in X$ there exists $\lim _{r \rightarrow \infty} C(r)=: P x$, then Theorem 2 and Remarks 2 and 3 imply that $P x \in \operatorname{Fix}(T(\cdot))$ and $P x \in \overline{\operatorname{co}\{T(t) x: 0 \leq t<\infty\}}$.

(ii) It follows from part (i) that if $\bar{T}$ is mean ergodic and satisfies (13) (resp., condition (iii) of Remark 3), then $\operatorname{Im} P \subseteq \operatorname{Fix}(T(\cdot)$ ), where

$$
P:=\tau_{s}-\lim _{r \rightarrow \infty} C(r) .
$$

On the other hand, $C(r) x=x$ for every $x \in \operatorname{Fix}(T(\cdot))$ and hence, $x=P x$. Thus

$$
\operatorname{Im} P=\operatorname{Fix}(T(\cdot)) .
$$

Moreover, by [21, Lemma 1.8, p.78] we have

$$
\text { Ker } P=\overline{\operatorname{span}\{x-T(t) x: t \geq 0, x \in X\}} \text {. }
$$

Note that the completeness requirement of $X$ in [21, Lemma 1.8, p.78] is not actually needed in the proof and that the equicontinuity requirement of $\{C(r)\}_{r \geq 0}$ is needed only to show the closedness of $\operatorname{Ker} P$. This is non-trivial in the more general situation considered in [21] because $P$ there is neither necessarily continuous nor defined on the whole space $X$. Of course, if $T$ is assumed to be mean ergodic, then the closedness of Ker $P$ follows directly from this hypothesis.

Next, let $y_{1}, y_{2} \in \overline{\operatorname{co}\{T(t) x: 0 \leq t<\infty\}} \cap \operatorname{Fix}(T(\cdot))$ for some fixed $x \in X$. It follows that

$$
y_{1}-y_{2}=\left(y_{1}-x\right)-\left(y_{2}-x\right) \in \overline{\operatorname{span}\{z-T(t) z: t \geq 0, z \in X\}} .
$$


Thus, (16) implies that $P\left(y_{1}-y_{2}\right)=0$. On the other hand, $y_{1}, y_{2} \in \operatorname{Fix}(T(\cdot))$ implies that $C(r)\left(y_{1}-y_{2}\right)=y_{1}-y_{2}$ for every $r \geq 0$. Hence, $0=P\left(y_{1}-\right.$ $\left.y_{2}\right)=y_{1}-y_{2}$. This, together with (15), shows that for every $x \in X$ the set $\operatorname{co}\{T(t) x: 0 \leq t<\infty\} \cap \operatorname{Fix}(T(\cdot))$ contains exactly one element, i.e., $P x$. Therefore, by [9, Lemma 4.1] we obtain that $P$ is a projection as well as $P S=$ $S P=P$ for every $S \in \mathcal{L}(X)$ with the property that $S y \in \overline{\operatorname{co}\{T(t) y: 0 \leq t<\infty\}}$ for all $y \in X$.

(iii) If $T$ is a mean ergodic, locally equicontinuous $C_{0}$-semigroup, then $\{C(r) x\}_{r \geq 0}$ is relatively sequentially compact for each $x \in X$ via the continuity of the map $r \mapsto C(r) x$ in $[0, \infty)$ (cf. Lemma 1(vi)) and the mean ergodicity of $T$.

(iv) If $T$ is a mean ergodic, locally equicontinuous $C_{0}$-semigroup, then necessarily condition (iii) in Remark 3 is satisfied. Indeed, by changing variables and applying the semigroup law we have

$$
\begin{aligned}
C(r+t) & =\frac{1}{r+t} \int_{0}^{r} T(s) d s+\frac{1}{r+t} \int_{r}^{r+t} T(s) d s \\
& =\frac{r}{r+t} C(r)+\frac{1}{r+t} \int_{0}^{t} T(r+s) d s \\
& =\frac{r}{r+t}\left[C(r)+\frac{T(r)}{r} t C(t)\right], \quad r, t>0 .
\end{aligned}
$$

By the mean ergodicity of $T$, it follows from (17) that, for any $t>0$ fixed, $\frac{T(r)}{r} C(t)=\frac{1}{t} \frac{T(r)}{r} t C(t) \rightarrow 0$ in $\mathcal{L}_{s}(X)$ as $r \rightarrow \infty$.

Remark 5 (i) As noted prior to Lemma 1, equicontinuity of $(T(t))_{t \geq 0}$ implies equicontinuity of $\{C(r)\}_{r \geq 0}$. Also, (5) implies that (13) is satisfied.

(ii) If $X$ is barrelled and $(T(t))_{t>0}$ is a strongly continuous $C_{0}$-semigroup in $X$, then mean ergodicity of $(T(t))_{t \geq 0}$ implies that $\{C(r)\}_{r \geq 0}$ is equicontinuous. That is, $\{C(r)\}_{r \geq 0}$ satisfies (E3).

(iii) Let $X$ be a sequentially complete $\mathrm{lcHs}$ and $(T(t))_{t \geq 0}$ be a locally equicontinuous $C_{0}$-semigroup on $X$. Then

$\operatorname{Ker} A=\operatorname{Fix}(T(\cdot))$ and $\overline{\operatorname{Im} A}=\overline{\operatorname{span}\{x-T(t) x: t \geq 0, x \in X\}}$,

where $(A, D(A))$ is the infinitesimal generator of $(T(t))_{t \geq 0}$. The first identity in (18) follows from the definition of the infinitesimal generator and (6).

Let $x \in D(A)$ and set $y:=A x$, i.e., $y=\lim _{t \rightarrow 0^{+}}(T(t) x-x) / t$ in $X$. Then, $y \in \overline{\operatorname{span}\{x-T(t) x: t \geq 0, x \in X\}}$. Conversely, let $z=x-T(t) x$ for some $x \in X, t>0$. As $D(A)$ is dense in $X$, there is a net $\left(x_{\alpha}\right)_{\alpha \in J} \subseteq D(A)$ with $x_{\alpha} \rightarrow x$ in $X$. By continuity, the net $(I-T(t)) x_{\alpha}=x_{\alpha}-T(t) x_{\alpha} \rightarrow z$ in $X$. Now $x_{\alpha} \in D(A)$ and so $A T(s) x_{\alpha} \in \operatorname{Im} A$ for $s \in[0, t]$. By properties of the Riemann integral, $\int_{0}^{t} A T(s) x_{\alpha} d s \in \overline{\operatorname{Im} A}$. Then (6) implies $x_{\alpha}-T(t) x_{\alpha}=$ $-\int_{0}^{t} A T(s) x_{\alpha} d s \in \overline{\operatorname{Im} A}$, for $\alpha \in J$. Passing to the limit we obtain $z \in \overline{\operatorname{Im} A}$.

(iv) The identity $\operatorname{Ker} A=\operatorname{Fix}(T(\cdot))$ in (18) is valid even if $X$ is not sequentially complete and $(T(t))_{t \geq 0}$ is only a strongly continuous $C_{0}$-semigroup (not 
necessarily locally equicontinuous). Indeed, for $x \in X$ and $t>0$, the formulae (6) remain valid under these weaker hypotheses; see Proposition 1.2 and its proof in [18].

(v) Let $X$ be a sequentially complete lcHs and $(T(t))_{t>0} \subseteq \mathcal{L}(X)$ be a locally equicontinuous $C_{0}$-semigroup on $X$. If (13) is satisfied and $\{C(r)\}_{r \geq 0}$ is equicontinuous, then $\{C(r)\}_{r \geq 0}$ is a $T$-ergodic net. Arguing as in the proof of [21, p.78, Lemma 1.8], where the completeness requirement on $X$ is not actually needed, yields the analogous fact from there that

$$
\overline{\operatorname{span}\{x-T(t) x: t \geq 0, x \in X\}}=\left\{x \in X: \lim _{r \rightarrow \infty} C(r) x=0\right\} .
$$

(vi) Recall that $y \in Y$ is a cluster point of the net $\left\{u_{\alpha}\right\}$ in a topological space $Y$ if, for each neighbourhood $\mathcal{U}$ of $y$ and each $\beta$, there is $\alpha \geq \beta$ such that $u_{\alpha} \in \mathcal{U}$. A subset $A \subseteq Y$ is called relatively countably compact if every sequence in $A$ has a cluster point in $Y$. So, if the set $\{C(r) x\}_{r \geq 0}$ is relatively countably $\sigma\left(X, X^{\prime}\right)$-compact for some $x \in X$, then $\{C(r) x\}_{r \geq 0}$ has a cluster point in $X_{\sigma}$. Indeed, if $r_{n} \uparrow \infty$, then the sequence $\left\{C\left(r_{n}\right) x\right\}_{n=1}^{\infty}$ has a cluster point $y$ in $X_{\sigma}$. Let $\mathcal{U}$ be a neighbourhoood of $y$ in $X_{\sigma}$ and $R \in[0, \infty)$. Select $N \in \mathbb{N}$ satisfying $r_{N}>R$. As $y$ is a cluster point of $\left\{C\left(r_{n}\right) x\right\}_{n=1}^{\infty}$ in $X_{\sigma}$ there is $n>N$ such that $C\left(r_{n}\right) x \in \mathcal{U}$. Then $r_{n}>R$ and we can conclude that $y$ is also a cluster point of $\{C(r) x\}_{r \geq 0}$ in $X_{\sigma}$.

For Banach spaces $X$ the formulae (18) are known, [11, p.278], [21, p.83].

Collecting various facts from above yields the following result.

Proposition 3 Let $X$ be a sequentially complete lcHs and $(T(t))_{t \geq 0} \subset \mathcal{L}(X)$ be a locally equicontinuous $C_{0}$-semigroup on $X$. Suppose that the following three conditions are satisfied.

(i) Condition (13) is satisfied, i.e., $\frac{T(t)}{t} \rightarrow 0$ in $\mathcal{L}_{s}(X)$ as $t \rightarrow \infty$.

(ii) $\{C(r)\}_{r>0}$ is equicontinuous.

(iii) The net

$$
\{C(r) x\}_{r \geq 0} \text { is relatively countably } \sigma\left(X, X^{\prime}\right) \text {-compact, } \forall x \in X .
$$

Then $(T(t))_{t \geq 0}$ is mean ergodic and the limit $P=\tau_{s}-\lim _{r \rightarrow \infty} C(r)$ is a projection.

Proof Conditions (i), (ii) imply that $\{C(r) x\}_{r \geq 0}$ is a $T$-ergodic net; cf. Remarks 3 and Remark 5(v). So, the result follows from Theorem 2 and Remark 4(ii) (via Remark 5(vi)).

Remark 6 Regarding Proposition 3, let $X$ be a sequentially complete lcHs and $(T(t))_{t \geq 0} \subset \mathcal{L}(X)$ a locally equicontinuous $C_{0}$-semigroup.

(i) In a lcHs $X$ all relatively $\sigma\left(X, X^{\prime}\right)$-compact sets and all relatively sequentially $\sigma\left(X, X^{\prime}\right)$-compact sets are necessarily relatively countably $\sigma\left(X, X^{\prime}\right)$ compact. These are the only implications between these three notions which hold in general. All three notions coincide whenever $X_{\sigma}$ is angelic, [12, p.31]. A 
large list of spaces $X$ for which $X_{\sigma}$ is angelic occurs in [12, §3.10]. In particular, $X_{\sigma}$ is angelic for all Fréchet (hence, all Banach) spaces $X$.

So, if $(T(t))_{t>0}$ is mean ergodic, then Remark 4(iii) implies that (19) holds.

(ii) If $X$ is barrelled, then (iii) implies (ii). Indeed, relatively countably $\sigma\left(X, X^{\prime}\right)$-compact sets are bounded, $\left[19, \S 24\right.$, p.310]. So, (iii) ensures $\{C(r)\}_{r \geq 0}$ is bounded in $\mathcal{L}_{s}(X)$ and hence, equicontinuous ( $X$ is barrelled), [20, p.137]. That is, (ii) holds.

(iii) Let $X$ be barrelled. Then conditions (i) and (iii) of Proposition 3 alone imply that $(T(t))_{t>0}$ is mean ergodic. Indeed, (ii) is then automatic (by (ii)).

(iv) Suppose (i), (ii) of Proposition 3 hold. Then $\{C(r)\}_{r \geq 0}$ is a $T$-ergodic net, cf. Remarks 3 and 5(v). As observed in Remark 5(v) the completeness of $X$ is not needed in Lemma 1.8 of [21, p.78]. Then an examination of the proof of part (b) of the Koliha, Nagel, Sato Theorem, [21, Theorem 1.9, p.79], reveals that also there $X$ is not required to be complete and we can conclude

Fact. $(T(t))_{t \geq 0}$ is mean ergodic if and only if Fix $(T(\cdot))$ separates $\operatorname{Fix}\left(T(\cdot)^{\prime}\right)$.

A locally equicontinuous $C_{0}$-semigroup $(T(t))_{t>0} \subseteq \mathcal{L}(X)$ is called weakly mean ergodic if $\lim _{r \rightarrow \infty} C(r)=P$ exists in $\mathcal{L}_{s}\left(X_{\sigma}\right)$

Corollary 1 Let $X$ be a sequentially complete lcHs and $(T(t))_{t \geq 0} \subseteq \mathcal{L}(X)$ be an equicontinuous $C_{0}$-semigroup. The following assertions are equivalent.

(i) $(T(t))_{t \geq 0}$ is mean ergodic.

(ii) $(T(t))_{t \geq 0}$ is weakly mean ergodic.

(iii) Condition (19) is satisfied.

Proof (i) $\Rightarrow$ (ii) is clear.

(i) $\Rightarrow$ (iii). This was pointed out in Remerk $6(\mathrm{i})$.

(iii) $\Rightarrow$ (i). This follows from Proposition 3 and Remarks 4(ii), 5(i).

(ii) $\Rightarrow$ (iii). Lemma 1 (vi) implies that $r \mapsto C(r)$ is continuous from $[0, \infty)$ into $\mathcal{L}_{s}\left(X_{\sigma}\right)$. Fix $x \in X$ and let $\left\{C\left(r_{n}\right) x\right\}_{n=1}^{\infty}$ be any sequence in $\{C(r) x\}_{r \geq 0}$. If there exists $M>0$ with $r_{n} \leq M$ for all $n \in \mathbb{N}$, then the continuity of $C(\cdot) x:[0, M] \rightarrow X_{\sigma}$ implies that $\{C(r) x\}_{r \in[0, M]}$ is $\sigma\left(X, X^{\prime}\right)$-compact and so $\left\{C\left(r_{n}\right) x\right\}_{n=1}^{\infty}$ has a cluster point in $X_{\sigma}$. Otherwise there exists a sequence $r_{n(k)} \uparrow \infty$ in which case $\lim _{k \rightarrow \infty} C\left(r_{n(k)}\right) x=y$ exists in $X_{\sigma}$. Hence, $y$ is a cluster point of $\left\{C\left(r_{n}\right) x\right\}_{n=1}^{\infty}$ in $X_{\sigma}$; argue along the lines of Remark 5(vi). So, (19) holds for $\{C(r) x\}_{r \geq 0}$.

Recall that a lcHs $X$ is semi-reflexive (resp. semi-Montel) if and only if every bounded subset of $X$ is relatively $\sigma\left(X, X^{\prime}\right)$-compact (resp. relatively compact), [24, Proposition 23.18].

Corollary 2 Let $X$ be a lcHs and $(T(t))_{t \geq 0} \subseteq \mathcal{L}(X)$ an equicontinuous $C_{0}-$ semigroup.

(i) If $X$ is semi-reflexive, then $(T(t))_{t \geq 0}$ is mean ergodic.

(ii) If $X$ is semi-Montel, then $(T(t))_{t \geq 0}$ is uniformly mean ergodic. 
Proof (i) The space $X$ is necessarily quasicomplete, [15, p.229], and by Remark 5(i) the net $\{C(r)\}_{r \geq 0}$ is equicontinuous. So, for each $x \in X$, the set $\{C(r) x\}_{r \geq 0} \in \mathcal{B}(X)$, i.e., $\{C(r) x\}_{r \geq 0}$ is relatively $\sigma\left(X, X^{\prime}\right)$-compact. Then Remark 6(i) shows that (19) holds and so the mean ergodicity of $(T(t))_{t \geq 0}$ follows from Corollary 1.

(ii) As $X$ is quasicomplete and semi-reflexive, [15, Propositions 11.5.1 \& 11.5.2], $(T(t))_{t \geq 0}$ is mean ergodic by part (i), i.e., $P:=\lim _{r \rightarrow \infty} C(r)$ exists in $\mathcal{L}_{s}(X)$. Since $\{C(r)\}_{r \geq 0}$ is equicontinuous (see Remark $5(\mathrm{i})$ ), also $H:=$ $\{P\} \cup\{C(r)\}_{r \geq 0}$ is equicontinuous. So, $\tau_{s}$ and the topology $\tau_{c}$ in $\mathcal{L}(X)$ of uniform convergence on precompact subsets of $X$ coincide on $H$, [20, (2) p.139]. But, bounded sets in $X$ are relatively compact (so, $\tau_{c}=\tau_{b}$ ). Hence, $C(r) \rightarrow P$ in $\mathcal{L}_{b}(X)$ as $r \rightarrow \infty$, i.e., $(T(t))_{t \geq 0}$ is uniformly mean ergodic.

An individual operator $T \in \mathcal{L}(X)$ is mean ergodic (resp. uniformly mean ergodic) if its Cesàro means $\left\{T_{[n]}\right\}_{n=1}^{\infty}$ (cf. (1)) converge in $\mathcal{L}_{s}(X)$ (resp. in $\left.\mathcal{L}_{b}(X)\right)$.

Theorem 4 Let $X$ be a sequentially complete lcHs and $(T(t))_{t \geq 0} \subseteq \mathcal{L}(X)$ be a locally equicontinuous $C_{0}$-semigroup such that $\{C(r)\}_{r \geq 0}$ is equicontinuous.

(i) If $T\left(t_{0}\right)$ is mean ergodic for some $t_{0}>0$, then $(T(t))_{t \geq 0}$ is mean ergodic.

(ii) If $T\left(t_{0}\right)$ is uniformly mean ergodic for some $t_{0}>0$, then $(T(t))_{t \geq 0}$ is uniformly mean ergodic.

Proof (i) For any fixed $r>0$, set $n=\left[\frac{r}{t_{0}}\right]$ so that $r=t_{0}(n+\alpha)$ with $0 \leq \alpha<1$. Arguing as in Remark 4(iv) we obtain

$$
\begin{aligned}
C(r) & =t_{0} \frac{n}{r}\left[\frac{1}{n} \sum_{m=0}^{n-1}\left(T\left(t_{0}\right)\right)^{m} C\left(t_{0}\right)+\frac{T\left(t_{0} n\right)}{t_{0} n} t_{0} \alpha C\left(t_{0} \alpha\right)\right] \\
& =t_{0} \frac{n}{r}\left[T\left(t_{0}\right)_{[n]} C\left(t_{0}\right)+\frac{\left(T\left(t_{0}\right)\right)^{n}}{t_{0} n} t_{0} \alpha C\left(t_{0} \alpha\right)\right] .
\end{aligned}
$$

Fix $x \in X$. By continuity of $\alpha \mapsto t_{0} \alpha C\left(t_{0} \alpha\right) x$ the set $\left\{t_{0} \alpha C\left(t_{0} \alpha\right) x: 0 \leq \alpha \leq 1\right\}$ is compact in $X$. As $T\left(t_{0}\right)$ is mean ergodic, $\lim _{n \rightarrow \infty} T\left(t_{0}\right)_{[n]}$ exists in $\mathcal{L}_{s}(X)$. So, from

$$
\frac{\left(T\left(t_{0}\right)\right)^{n}}{n}=T\left(t_{0}\right)_{[n]}-\frac{(n-1)}{n} T\left(t_{0}\right)_{[n-1]}, \quad n \in \mathbb{N}
$$

(routine to verify), it follows $\tau_{s}-\lim _{n \rightarrow \infty} \frac{\left(T\left(t_{0}\right)\right)^{n}}{n}=0$. Accordingly,

$$
\frac{\left(T\left(t_{0}\right)\right)^{n}}{t_{0} n}\left(t_{0} \alpha C\left(t_{0} \alpha\right) x\right) \rightarrow 0, \text { as } n \rightarrow \infty
$$

uniformly for $\alpha \in[0,1]$. As $\frac{t_{0} n}{r}=1-\frac{t_{0} \alpha}{r} \rightarrow 1$ as $n \rightarrow \infty$ (i.e., as $r \rightarrow \infty$ ), by $(20)$ and (22) we can conclude that $C(r) x$ converges to some $P x$ in $X$ as $r \rightarrow \infty$. Since $x$ is arbitrary and $\{C(r)\}_{r \geq 0}$ is equicontinuous, we obtain that $P \in \mathcal{L}(X)$. 
(ii) Proceed as in (i) to deduce via $(21)$ that $\tau_{b}$ - $\lim _{n \rightarrow \infty} \frac{\left(T\left(t_{0}\right)\right)^{n}}{n}=0$. As

$$
t_{0} \frac{n}{r} \frac{\left(T\left(t_{0}\right)\right)^{n}}{t_{0} n} t_{0} \alpha C\left(t_{0} \alpha\right)=\frac{\left(T\left(t_{0}\right)\right)^{n}}{n}\left(\frac{t_{0} \alpha n}{r} C\left(t_{0} \alpha\right)\right)
$$

with $\frac{t_{0} \alpha n}{r} \in[0,1]$ and $t_{0} \alpha \in\left[0, t_{0}\right]$ for all $\alpha \in[0,1]$ and $r \geq 0$ we see that

$$
\left(t_{0} \frac{n}{r} \frac{\left(T\left(t_{0}\right)\right)^{n}}{t_{0} n} t_{0} \alpha C\left(t_{0} \alpha\right)\right)(B) \subseteq \frac{\left(T\left(t_{0}\right)\right)^{n}}{n}\left(\cup_{\substack{\beta \in[0,1] \\ s \in\left[0, t_{0}\right]}} \beta C(s)(B)\right)=: \frac{\left(T\left(t_{0}\right)\right)^{n}}{n}\left(B^{*}\right)
$$

for each $B \in \mathcal{B}(X)$. But, equicontinuity of $\{C(r)\}_{r \geq 0}$ implies that also $B^{*} \in$ $\mathcal{B}(X)$ and hence, $t_{0} \frac{n}{r} \frac{\left(T\left(t_{0}\right)\right)^{n}}{t_{0} n} t_{0} \alpha C\left(t_{0} \alpha\right) \rightarrow 0$ in $\mathcal{L}_{b}(X)$ as $n \rightarrow \infty$ uniformly for $\alpha \in[0,1]$. Furthermore, since $\frac{t_{0} n}{r} \in[0,1]$ for all $r \geq 0$ we see, for each $n \in \mathbb{N}$, that

$$
\left(t_{0} \frac{n}{r} T\left(t_{0}\right)_{[n]} C\left(t_{0}\right)\right)(B) \subseteq T\left(t_{0}\right)_{[n]} C\left(t_{0}\right)\left(\cup_{\gamma \in[0,1]} \gamma B\right)=: T\left(t_{0}\right)_{[n]}(D)
$$

with $D \in \mathcal{B}(X)$. So, also $t_{0} \frac{n}{r} T\left(t_{0}\right)_{[n]} C\left(t_{0}\right)$ converges in $\mathcal{L}_{b}(X)$ as $n \rightarrow \infty$. Then (20) implies $\lim _{r \rightarrow \infty} C(r)$ exists in $\mathcal{L}_{b}(X)$, i.e., $(T(t))_{t \geq 0}$ is uniformly mean ergodic.

Remark 7 Theorem 4 ensures an equicontinuous $C_{0}$-semigroup $(T(t))_{t \geq 0}$ in a sequentially complete lcHs $X$ is mean ergodic if there is $t_{0}>0$ such that $T\left(t_{0}\right)$ is mean ergodic. For results of this kind in Banach spaces we refer to [30], [32], and the references therein. The converse is false, both in Banach spaces, [11, p.345], [21, p.83], [32, Example (d), p.75], and also in (non-trivial) nonnormable Fréchet spaces. Indeed, let $Y:=\left\{f \in L^{1}(\mathbb{R}): \int_{\mathbb{R}} f(s) d s=0\right\}$. Then $Y$ is closed in $L^{1}(\mathbb{R})$ and hence, $\left(Y,\|\cdot\|_{1}\right)$ is a Banach space. The left translation semigroup $(T(t))_{t \geq 0}$ on $Y$ is an equicontinuous $C_{0}$-semigroup which is mean ergodic, but $\left\{T\left(t_{0}\right)_{[n]}\right\}_{n=1}^{\infty}$ fails to converge for every $t_{0}>0,[11, \mathrm{Ch} . \mathrm{V}, 4.13 .3]$. The space $X:=Y^{\mathbb{N}}$ endowed with the product topology is a non-normable Fréchet space and $S(t) f:=\left(T(t) f_{n}\right)_{n}$, for $f=\left(f_{n}\right)_{n} \in X, t \geq 0$, defines an equicontinuous $C_{0}$-semigroup in $X$ which is mean ergodic but, for no $t_{0}>0$ is $S\left(t_{0}\right)$ mean ergodic.

A sequence $\left(x_{n}\right)_{n \in \mathbb{N}}$ in a lcHs $X$ is called a basis if, for every $x \in X$, there is a unique sequence $\left(\alpha_{n}\right)_{n \in \mathbb{N}} \subseteq \mathbb{C}$ such that the series $\sum_{n=1}^{\infty} \alpha_{n} x_{n}$ converges to $x \in X$. By setting $f_{n}(x):=\alpha_{n}$ we obtain a linear form $f_{n}: X \rightarrow \mathbb{C}$. If $\left(f_{n}\right)_{n \in \mathbb{N}} \subseteq X^{\prime}$, then $\left(x_{n}\right)_{n \in \mathbb{N}}$ is called a Schauder basis for $X$.

The next result extends its Banach space counterpart, [26, Theorem 3.4].

Theorem 5 Let $X$ be a complete, barrelled lcHs with a Schauder basis. Then the following assertions are equivalent.

(i) $X$ is reflexive.

(ii) Every equicontinuous $C_{0}$-semigroup on $X$ is mean ergodic.

(iii) Every equicontinuous, uniformly continuous $C_{0}$-semigroup on $X$ is mean ergodic. 
Proof (i) $\Rightarrow($ ii). As a reflexive space is semi-reflexive, Corollary 2(i) implies (ii).

(ii) $\Rightarrow$ (iii). Obvious.

(iii) $\Rightarrow(\mathrm{i})$. Suppose (i) fails, i.e., $X$ is not reflexive. By [1, Theorem 1.2] the space $X$ admits a non-shrinking basis. So, there is a power bounded operator $A \in \mathcal{L}(X)$ (i.e., $\left\{A^{n}\right\}_{n=1}^{\infty}$ is equicontinuous) which is not mean ergodic, $[3$, Theorem 3.4].

As $A$ is power bounded, given $p \in \Gamma_{X}$ there exist $M_{p}>0$ and $q \in \Gamma_{X}$ such that $p\left(A^{n} x\right) \leq M_{p} q(x)$ for all $x \in X, n \in \mathbb{N}$. So, the seminorms $\left\{\bar{p}: p \in \Gamma_{X}\right\}$ given by $\bar{p}(x):=\sup _{n \geq 0} p\left(A^{n} x\right)$ also generate the lc-topology of $X$ because $p(x) \leq \bar{p}(x) \leq M_{p} q(x) \leq M_{p} \bar{q}(x)$, for $x \in X, p \in \Gamma_{X}$. Moreover,

$$
\bar{p}\left(A^{n} x\right)=\sup _{m \geq 0} p\left(A^{m} A^{n} x\right)=\sup _{h \geq n} p\left(A^{h} x\right) \leq \bar{p}(x), \quad x \in X, p \in \Gamma_{X} .
$$

So, given $t \geq 0$, the operator $T(t):=\sum_{k=0}^{\infty} \frac{t^{k} A^{k}}{k !}$ exists in $\mathcal{L}(X)$ and satisfies

$$
\bar{p}(T(t) x) \leq e^{t} \bar{p}(x), \quad x \in X, p \in \Gamma_{X}
$$

[33, p.245]. Moreover, $(T(t))_{t \geq 0}$ is a uniformly continuous $C_{0}$-semigroup with $(A, X)$ as infinitesimal generator. Indeed, uniform continuity follows via (23), which implies for $p \in \Gamma_{X}$ and $B \in \mathcal{B}(X)$ that $\sup _{x \in B} \bar{p}((T(t)-I) x) \leq$ $K\left(e^{t}-1\right)$, for $t \geq 0$, where $K:=\sup _{x \in B} \bar{p}(x)$. Then $(24)$ shows that $S(t):=$ $e^{-t} T(t)$, for $t \geq 0$, is an equicontinuous, uniformly continuous $C_{0}$-semigroup on $X$ with $(A-I, X)$ as infinitesimal generator. Now, Remark 5(iii) implies (see (18)) that $\operatorname{Ker}(A-I)=\operatorname{Fix}(S(\cdot))$. Observe the $\mathrm{lcHs} X_{\sigma^{*}}^{\prime}$ is quasicomplete, [19, §27]. Moreover, $r \mapsto S(r)^{\prime}$, for $r \geq 0$, is a $C_{0}$-semigroup in $\mathcal{L}\left(X_{\sigma^{*}}^{\prime}\right)$ with infinitesimal generator $\left(A^{\prime}, X^{\prime}\right),[18, \S 2 \&$ Proposition 2.1]. Actually, $\left(S(r)^{\prime}\right)_{r \geq 0}$ is an equicontinuous, uniformly continuous semigroup in $\mathcal{L}\left(X_{\beta}^{\prime}\right)$ because $S(r)^{\prime} \in \mathcal{L}\left(X_{\beta}^{\prime}\right)$, with $S(r)^{\prime}=e^{-r} \sum_{k=1}^{\infty} \frac{r^{k}\left(A^{\prime}\right)^{k}}{k !}$ for $r \geq 0$, and $A^{\prime} \in \mathcal{L}\left(X_{\beta}^{\prime}\right)$ is power bounded. As $X_{\beta}^{\prime}$ is quasicomplete, [15, Proposition 11.2.4, p.222], Remark 5(iii) yields $\operatorname{Ker}\left(A^{\prime}-I\right)=\operatorname{Fix}\left(S(\cdot)^{\prime}\right)$. By hypothesis, the equicontinuous, uniformly continuous semigroup $(S(t))_{t \geq 0} \subseteq \mathcal{L}(X)$ is mean ergodic. Equicontinuity of $(S(t))_{t>0}$ implies the hypotheses of Remark $6($ iv $)$ are satisfied. So, Fix $(S(\cdot))$ separates Fix $\left(S(\cdot)^{\prime}\right)$, i.e., $\operatorname{Ker}(A-I)$ separates $\operatorname{Ker}\left(A^{\prime}-I\right)$. An examination of the proof of Sine's Theorem for an individual operator in a Banach space, as given in [21, p.74], shows it is based purely on a duality argument and so carries over to lcHs', [27, Theorem 13], thereby allowing us to conclude that $X=\operatorname{Ker}(A-I) \oplus \overline{\operatorname{Im}(A-I)}$. As $A$ is power bounded, we have $\overline{\operatorname{Im}(A-I)}=\left\{x \in X: \lim _{n \rightarrow \infty} A_{[n]} x=0\right\}$, [33, p.213]. Given $x \in X$, write $x=u+v$ with $u \in \operatorname{Ker}(A-I)$ and $v \in \overline{\operatorname{Im}(A-I)}$. Then $A_{[n]} x=u+A_{[n]} v$, for $n \in \mathbb{N}$, and so $\lim _{n \rightarrow \infty} A_{[n]} x=u$ exists in $X$. As $X$ is barrelled, the limit operator $x \mapsto \lim _{n \rightarrow \infty} A_{[n]} x$ belongs to $\mathcal{L}(X)$, i.e., $A$ is mean ergodic. This contradicts the choice of $A$ and so $X$ must be reflexive. 


\section{Semigroups and mean ergodicity in GDP-spaces}

In Banach GDP-spaces every strongly continuous $C_{0}$-semigroup is uniformly continuous, [22, Theorem 6], [23, Theorem 3]. This also holds for exponentially equicontinuous $C_{0}$-semigroups acting in Fréchet GDP-spaces which, additionally, are also quojections, [4, Theorem 3.5]. In this section we extend this fact to quasicomplete, barrelled, GDP-spaces and to $C_{0}$-semigroups which are only locally equicontinuous. The methods introduced also allow us to characterize mean ergodicity of $C_{0}$-semigroups $(T(t))_{t \geq 0}$ satisfying (13) and acting in complete, barrelled, Grothendieck spaces in terms of the annihilator $(\operatorname{Fix}(T(\cdot)))^{\perp}$. If, additionally, $(T(t))_{t \geq 0}$ is uniformly mean ergodic, then $\left(T(s)^{\prime}\right)_{s \geq 0}$ is mean ergodic in $X_{\beta}^{\prime}$.

A lcHs $X$ is a Grothendieck space if sequences in $X^{\prime}$ which are convergent for $\sigma\left(X^{\prime}, X\right)$ also converge for $\sigma\left(X^{\prime}, X^{\prime \prime}\right)$. Reflexive lcHs' are Grothendieck spaces. A lcHs $X$ has the Dunford-Pettis property (briefly, DP) if every element of $\mathcal{L}(X, Y)$, for $Y$ any quasicomplete lcHs, which transforms elements of $\mathcal{B}(X)$ into relatively $\sigma\left(Y, Y^{\prime}\right)$-compact subsets of $Y$, also transforms $\sigma\left(X, X^{\prime}\right)$ compact subsets of $X$ into relatively compact subsets of $Y$, [10, pp.633-634]. It suffices if $Y$ runs through all Banach spaces, [5, p.79]. A reflexive lcHs has the DP property if and only if it is Montel, [10, p.634]. A Grothendieck lcHs $X$ with the DP property is called a GDP-space. Every Montel lcHs is a GDP-space, [5, Remark 2.2], [2, Corollary 3.8]. For examples of Banach GDP-spaces, see [22], [23].

Proposition 6 Let $X$ be a quasicomplete, barrelled lcHs and $(T(t))_{t \geq 0} \subseteq$ $\mathcal{L}(X)$ be a semigroup.

(i) If $(T(t))_{t \geq 0}$ is a strongly continuous $C_{0}$-semigroup and $X$ is a Grothendieck space, then $\left(T(s)^{\prime}\right)_{s \geq 0}$ is a locally equicontinuous $C_{0}$-semigroup in $X_{\beta}^{\prime}$.

(ii) If $\left(T(s)^{\prime \prime}\right)_{s \geq 0}$ is a strongly continuous $C_{0}$-semigroup on $X_{\beta}^{\prime \prime}$, then $\left(T(s)^{\prime}\right)_{s \geq 0}$ is a locally equicontinuous $C_{0}$-semigroup on $X_{\beta}^{\prime}$.

Proof (i) It is known that $\left(T(s)^{\prime}\right)_{s \geq 0} \subseteq \mathcal{L}\left(X_{\beta}^{\prime}\right)$, [20, p.134], and routine to check that $\left(T(s)^{\prime}\right)_{s \geq 0}$ is a semigroup. To verify that $\left(T(s)^{\prime}\right)_{s \geq 0}$ is locally equicontinuous fix $K>0$ and $B \in \mathcal{B}(X)$. By Remark $1(\mathrm{i}),(T(t))_{t \geq 0}$ is locally equicontinuous and so $C:=\{T(t) x: t \in[0, K], x \in B\} \in \mathcal{B}(X)$. Accordingly, for each $x^{\prime} \in X^{\prime}$,

$$
\sup _{s \in[0, K]} p^{B}\left(T(s)^{\prime} x^{\prime}\right)=\sup _{s \in[0, K]} \sup _{x \in B}\left|\left\langle x, T(s)^{\prime} x^{\prime}\right\rangle\right| \leq \sup _{y \in C}\left|\left\langle y, x^{\prime}\right\rangle\right|=p^{C}\left(x^{\prime}\right),
$$

which establishes the locally equicontinuity of $\left(T(s)^{\prime}\right)_{s \geq 0} \subseteq \mathcal{L}\left(X_{\beta}^{\prime}\right)$.

Applying Proposition 1 to $\left(T(s)^{\prime}\right)_{s \geq 0}$ in $X_{\beta}^{\prime}$ the proof is complete $\left(X_{\beta}^{\prime}\right.$ is quasicomplete being the strong dual of a barrelled space, [15, Proposition 11.2.4]), provided we verify $s \mapsto\left\langle T(s)^{\prime} x^{\prime}, x^{\prime \prime}\right\rangle$ is continuous on $[0, \infty)$, for $x^{\prime} \in X^{\prime}, x^{\prime \prime} \in X^{\prime \prime}$. Let $x^{\prime} \in X^{\prime}$. Fix $s^{*}>0$ and let $\left(s_{n}\right)_{n=1}^{\infty} \subseteq[0, \infty)$ converge to $s^{*}$. We need to check $T\left(s_{n}\right)^{\prime} x^{\prime} \rightarrow T\left(s^{*}\right)^{\prime} x^{\prime}$ in $\left(X^{\prime}, \sigma\left(X^{\prime}, X^{\prime \prime}\right)\right)$ as $n \rightarrow \infty$. Since 
$X$ is a Grothendieck space, it suffices to prove $\left\langle x, T\left(s_{n}\right)^{\prime} x^{\prime}\right\rangle \rightarrow\left\langle x, T\left(s^{*}\right)^{\prime} x^{\prime}\right\rangle$, for $x \in X$. But, this is so as

$$
\left|\left\langle x, T\left(s_{n}\right)^{\prime} x^{\prime}\right\rangle-\left\langle x, T\left(s^{*}\right)^{\prime} x^{\prime}\right\rangle\right|=\left|\left\langle\left(T\left(s_{n}\right)-T\left(s^{*}\right)\right) x, x^{\prime}\right\rangle\right|, \quad n \in \mathbb{N},
$$

and the right-side converges to 0 as $n \rightarrow \infty$ because $(T(t))_{t \geq 0}$ is a strongly continuous $C_{0}$-semigroup. For $s^{*}=0$ we take $\left(s_{n}\right)_{n=1}^{\infty} \subseteq[0, \infty)$ with $s_{n} \rightarrow 0^{+}$.

(ii) As $X$ is barrelled, it is a topological subspace of $X_{\beta}^{\prime \prime}$, [15, Proposition 11.2.2]. Also, $T(s)^{\prime \prime} x=T(s) x$ for $s \geq 0, x \in X$, implies $(T(t))_{t>0} \subseteq \mathcal{L}(X)$ is a strongly continuous $C_{0}$-semigroup which, by Remark $1(\mathrm{i})$, is locally equicontinuous. Arguing as in (i) we conclude $\left(T(s)^{\prime}\right)_{s \geq 0} \subseteq \mathcal{L}\left(X_{\beta}^{\prime}\right)$ is a locally equicontinuous semigroup.

The $C_{0}$-property again follows from Proposition 1 applied to $\left(T(s)^{\prime}\right)_{s \geq 0}$ in $X_{\beta}^{\prime}$. Indeed, fix $s^{*}>0, x^{\prime} \in X^{\prime}$ and let $s_{n} \rightarrow s^{*}$ in $[0, \infty)$. Then, for $x^{\prime \prime} \in X^{\prime \prime}$, we have

$$
\left|\left\langle T\left(s_{n}\right)^{\prime} x^{\prime}, x^{\prime \prime}\right\rangle-\left\langle T\left(s^{*}\right)^{\prime} x^{\prime}, x^{\prime \prime}\right\rangle\right|=\left|\left\langle x^{\prime},\left(T\left(s_{n}\right)^{\prime \prime}-T\left(s^{*}\right)^{\prime \prime}\right) x^{\prime \prime}\right\rangle\right|, \quad n \in \mathbb{N},
$$

with the right-side converging to 0 as $n \rightarrow \infty$ because $\left(T(s)^{\prime \prime}\right)_{s \geq 0}$ is a strongly continuous $C_{0}$-semigroup in $X_{\beta}^{\prime \prime}$. So, $T\left(s_{n}\right)^{\prime} x^{\prime} \rightarrow T\left(s^{*}\right)^{\prime} x^{\prime}$ in $\left(\bar{X}^{\prime}, \sigma\left(X^{\prime}, X^{\prime \prime}\right)\right)$. For $s^{*}=0$ we take $\left(s_{n}\right)_{n=1}^{\infty} \subseteq[0, \infty)$ with $s_{n} \rightarrow 0^{+}$.

Lemma 2 Let $X$ be a lcHs with $(T(t))_{t \geq 0} \subseteq \mathcal{L}(X)$ a locally equicontinuous semigroup. If $\tau_{b}-\lim _{t \rightarrow 0^{+}}(T(t)-I)^{2}=0$, then $(T(t))_{t \geq 0}$ is uniformly continuous.

Proof Fix $B \in \mathcal{B}(X)$ and $q \in \Gamma_{X}$. By local equicontinuity, $\sup _{t \in[0,1]} q_{B}(T(t)-$ $I)<\infty$ and so $s_{q, B}:=\lim \sup _{t \rightarrow 0^{+}} q_{B}(T(t)-I)<\infty$. Then $2(T(t)-I)=$ $T(2 t)-I-(T(t)-I)^{2}$ implies, for $x \in B$ and $t \in[0,1 / 2]$, that

$$
2 q(T(t) x-x) \leq q((T(2 t)-I) x)+q\left((T(t)-I)^{2} x\right) \leq q((T(2 t)-I) x)+q_{B}\left((T(t)-I)^{2}\right) .
$$

Form the sup over $x \in B$ and let $t \rightarrow 0^{+}$gives $2 s_{q, B} \leq \lim \sup _{t \rightarrow 0^{+}} q_{B}(T(2 t)-$ $I) \leq s_{q, B}$. So, $s_{q, B}=0$ which implies $\lim _{t \rightarrow 0^{+}} T(t)=I$ in $\mathcal{L}_{b}(X)$.

Fix $t_{0}>0$. Then for $h>0$ and fixed $B \in \mathcal{B}(X), q \in \Gamma_{X}$, we have

$$
q_{B}\left(T\left(t_{0}+h\right)-T\left(t_{0}\right)\right)=q_{B}\left(T\left(t_{0}\right)(T(h)-I)\right)=q_{T\left(t_{0}\right) B}(T(h)-I) .
$$

Since $T\left(t_{0}\right) B \in \mathcal{B}(X)$, we have $\lim _{t \rightarrow t_{0}^{+}} T(t)=T\left(t_{0}\right)$ in $\mathcal{L}_{b}(X)$. By local equicontinuity, $C:=\left\{T(t) x: t \in\left[0, t_{0}\right], x \in B\right\} \in \mathcal{B}(X)$. So, for $h \in\left[-t_{0} / 2,0\right]$, we have

$$
q_{B}\left(T\left(t_{0}+h\right)-T\left(t_{0}\right)\right)=q_{B}\left(T\left(t_{0}+h\right)(I-T(-h))\right) \leq q_{C}(I-T(-h)),
$$

which implies also $\tau_{b}$ - $\lim _{t \rightarrow t_{0}^{-}} T(t)=T\left(t_{0}\right)$, i.e., $T:[0, \infty) \rightarrow \mathcal{L}_{b}(X)$ is continuous.

Theorem 7 Let $X$ be a barrelled, quasicomplete, GDP lcHs. Then every strongly continuous $C_{0}$-semigroup on $X$ is uniformly continuous. 
Proof Let $(T(t))_{t \geq 0}$ be a strongly continuous $C_{0}$-semigroup in $X$. Via Remark $1(\mathrm{i}),(T(t))_{t \geq 0}$ is locally equicontinuous. So, by Lemma 2 , it suffices to show

$$
\lim _{s \rightarrow 0^{+}} q_{B}\left((T(t)-I)^{2}\right)=0, \quad B \in \mathcal{B}(X), q \in \Gamma_{X} .
$$

If (25) does not hold, then there is $\varepsilon>0$, a sequence $s_{n} \downarrow 0$ in $[0, \infty)$, a bounded sequence $C:=\left(x_{n}\right)_{n=1}^{\infty} \subseteq X$, a seminorm $q \in \Gamma_{X}$, and a sequence $\left(x_{n}^{\prime}\right)_{n=1}^{\infty} \subseteq X^{\prime}$ satisfying $\left|\left\langle y, x_{n}^{\prime}\right\rangle\right| \leq q(y)$ for $y \in X, n \in \mathbb{N}$ such that, for all $n \in \mathbb{N}$, we have

$$
\varepsilon<\left|\left\langle\left(T\left(s_{n}\right)-I\right)^{2} x_{n}, x_{n}^{\prime}\right\rangle\right|=\left|\left\langle\left(T\left(s_{n}\right)-I\right) x_{n},\left(T\left(s_{n}\right)^{\prime}-I\right) x_{n}^{\prime}\right\rangle\right| .
$$

We first show $\left(T\left(s_{n}\right)^{\prime}-I\right) x_{n}^{\prime} \rightarrow 0$ in $\left(X^{\prime}, \sigma\left(X^{\prime}, X^{\prime \prime}\right)\right)$. As $X$ is a Grothendieck space, it suffices to show $\left\langle x,\left(T\left(s_{n}\right)^{\prime}-I\right) x_{n}^{\prime}\right\rangle \rightarrow 0$ as $n \rightarrow \infty$, for $x \in X$. But,

$$
\left|\left\langle x,\left(T\left(s_{n}\right)^{\prime}-I\right) x_{n}^{\prime}\right\rangle\right|=\left|\left\langle\left(T\left(s_{n}\right)-I\right) x, x_{n}^{\prime}\right\rangle\right| \leq q\left(\left(T\left(s_{n}\right)-I\right) x\right), \quad n \in \mathbb{N},
$$

which converges to 0 as $n \rightarrow \infty$ because $(T(t))_{t \geq 0}$ is a $C_{0}$-semigroup.

Proposition 6(i) implies $\left(T(s)^{\prime}\right)_{s \geq 0} \subseteq \mathcal{L}\left(X_{\beta}^{\prime}\right)$ is a locally equicontinuous, strongly continuous $C_{0}$-semigroup. As $C \in \mathcal{B}(X)$, we see from $\mid\left\langle\left(T\left(s_{n}\right)-\right.\right.$ $\left.I) x_{n}, x^{\prime}\right\rangle \mid \leq p^{C}\left(\left(T\left(s_{n}\right)^{\prime}-I\right) x^{\prime}\right)$, for $x^{\prime} \in X^{\prime}, n \in \mathbb{N}$, that $\left(T\left(s_{n}\right)-I\right) x_{n} \rightarrow 0$ in $X_{\sigma}$. But, $X$ has the DP-property and so Proposition 3.3(i) of [2] implies the right-side of (26) converges to 0 , which is impossible. So, (25) holds.

Remark 8 For $X$ a quojection GDP Fréchet space it was shown in [4, Theorem 3.5] that every exponentially equicontinuous $C_{0}$-semigroup in $X$ (locally equicontinuous and strongly continuous by Remark 1(ii), (iii)) is uniformly continuous with infinitesimal generator in $\mathcal{L}(X)$. Theorem 7 shows uniform continuity of the semigroup holds under milder conditions; metrizability of $X$ can be replaced by quasicompleteness and barrelledness and, even within the class of Fréchet spaces, $X$ need not be a quojection. However, there do exist nuclear Fréchet spaces (hence, GDP) and equicontinuous, uniformly continuous semigroups in such spaces whose infinitesimal generator is not in $\mathcal{L}(X)$, [4, Example 3.1]; such a space cannot be a quojection. For Banach spaces, Theorem 7 is due to H.P. Lotz, [22], [23].

In the Banach space setting the next result is proved in [23, Theorem 4].

Theorem 8 Let $X$ be a barrelled, quasicomplete lcHs with the DP-property and $(T(t))_{t \geq 0} \subseteq \mathcal{L}(X)$ be a semigroup such that $\left(T(s)^{\prime \prime}\right)_{s \geq 0}$ is a strongly continuous $C_{0}-$ semigroup on $X_{\beta}^{\prime \prime}$. Then $(T(t))_{t \geq 0}$ is uniformly continuous.

Proof From the proof of Proposition 6(ii) we see that $(T(t))_{t \geq 0}$ is a strongly continuous $C_{0}$-semigroup which is locally equicontinuous. We proceed via contradiction as in the proof of Theorem 7; our notation is from there. Set $D:=\left(x_{n}^{\prime}\right)_{n=1}^{\infty}$, which is equicontinuous in $X^{\prime}$. We need to show that

$$
\left(T\left(s_{n}\right)^{\prime}-I\right) x_{n}^{\prime} \rightarrow 0 \text { in }\left(X^{\prime}, \sigma\left(X^{\prime}, X^{\prime \prime}\right)\right), \text { for } n \rightarrow \infty,
$$


and also that

$$
\left(T\left(s_{n}\right)-I\right) x_{n} \rightarrow 0 \text { in }\left(X, \sigma\left(X, X^{\prime}\right)\right), \text { for } n \rightarrow \infty .
$$

Fix $x^{\prime \prime} \in X^{\prime \prime}$. Then, for $n \in \mathbb{N}$, we have $\left|\left\langle\left(T\left(s_{n}\right)^{\prime}-I\right) x_{n}^{\prime}, x^{\prime \prime}\right\rangle\right|=\mid\left\langle x_{n}^{\prime},\left(T\left(s_{n}\right)^{\prime \prime}-\right.\right.$ $\left.I) x^{\prime \prime}\right\rangle\left|\leq \sup _{u \in D}\right|\left\langle u,\left(T\left(s_{n}\right)^{\prime \prime}-I\right) x^{\prime \prime}\right\rangle \mid$. As $D \in \mathcal{B}\left(X_{\beta}^{\prime}\right)$ and $\left(T(s)^{\prime \prime}\right)_{s \geq 0}$ is a $C_{0^{-}}$ semigroup in $X_{\beta}^{\prime \prime}=\left(X_{\beta}^{\prime}\right)_{\beta}^{\prime}$, it follows $\lim _{n \rightarrow \infty} \sup _{u \in D}\left|\left\langle u,\left(T\left(s_{n}\right)^{\prime \prime}-\bar{I}\right) x^{\prime \prime}\right\rangle\right|=0$, i.e., (27) holds.

To verify (28), observe that $\left(T(s)^{\prime}\right)_{s \geq 0} \subseteq \mathcal{L}\left(X_{\beta}^{\prime}\right)$ is a locally equicontinuous, strongly continuous $C_{0}$-semigroup (cf. Proposition 6(ii)) and $X$ has the DPproperty. Hence, the argument in the proof of Theorem 7 can be repeated.

Turning our attention to mean ergodicity, we first require a preliminary result.

Lemma 3 Let $X$ be a sequentially complete lcHs and $(T(t))_{t>0} \subseteq \mathcal{L}(X)$ be a locally equicontinuous $C_{0}$-semigroup on $X$ which satisfies (13).

(i) If $(T(t))_{t \geq 0}$ is mean ergodic and $P \in \mathcal{L}(X)$ is the projection (14), then

$$
\operatorname{Im} P^{\prime}=\operatorname{Fix}\left(T(\cdot)^{\prime}\right) .
$$

(ii) Suppose that $X$ is complete and $\{C(r)\}_{r \geq 0} \subseteq \mathcal{L}(X)$ is equicontinuous. Then the following topological direct sum is a closed subspace of $X$ :

$$
\operatorname{Fix}(T(\cdot)) \oplus \overline{\operatorname{span}\{x-T(t) x: t \geq 0, x \in X\}} .
$$

Proof (i) Via Remark 4(ii), $P^{\prime} \in \mathcal{L}\left(X_{\beta}^{\prime}\right)$ satisfies $P^{\prime}=P^{\prime} T(s)^{\prime}=T(s)^{\prime} P^{\prime}$, for $s \geq 0$. In particular, $T(s)^{\prime} P^{\prime} x^{\prime}=P^{\prime} x^{\prime}$ for $s \geq 0, x^{\prime} \in X^{\prime}$, i.e., $P^{\prime} x^{\prime} \in \operatorname{Fix}\left(T(\cdot)^{\prime}\right)$ for $x^{\prime} \in X^{\prime}$. So, $\operatorname{Im} P^{\prime} \subseteq \operatorname{Fix}\left(T(\cdot)^{\prime}\right)$.

By Remark 1(iii), $(T(t))_{t \geq 0}$ is strongly continuous. Fix $x^{\prime} \in \operatorname{Fix}\left(T(\cdot)^{\prime}\right)$ so that $T(s)^{\prime} x^{\prime}=x^{\prime}$ for all $s \geq 0$. Then, for $r>0, x \in X$, we have $\left\langle x, C(r)^{\prime} x^{\prime}\right\rangle=$ $\left\langle\frac{1}{r} \int_{0}^{r} T(s) x d s, x^{\prime}\right\rangle=\frac{1}{r} \int_{0}^{r}\left\langle T(s) x, x^{\prime}\right\rangle d s=\frac{1}{r} \int_{0}^{r}\left\langle x, T(s)^{\prime} x^{\prime}\right\rangle d s=\left\langle x, x^{\prime}\right\rangle$, which implies $C(r)^{\prime} x^{\prime}=x^{\prime}$. For $x \in X$, it then follows from (14) that $\left\langle P x, x^{\prime}\right\rangle=$ $\lim _{r \rightarrow \infty}\left\langle C(r) x, x^{\prime}\right\rangle=\lim _{r \rightarrow \infty}\left\langle x, C(r)^{\prime} x^{\prime}\right\rangle=\left\langle x, x^{\prime}\right\rangle$ and hence, $\left\langle x, P^{\prime} x^{\prime}\right\rangle=$ $\left\langle x, x^{\prime}\right\rangle$, i.e., $P^{\prime} x^{\prime}=x^{\prime}$. So, $x^{\prime} \in \operatorname{Im} P^{\prime}$.

(ii) This is due to Koliha, Nagel and Sato, [21, Theorem 1.9(a), p.79].

We recall for a subspace $Y$ of a lcHs $X$ that its annihilator $Y^{\perp}:=\left\{x^{\prime} \in\right.$ $\left.X^{\prime}:\left\langle y, x^{\prime}\right\rangle=0 \forall y \in Y\right\}$ coincides with its polar $Y^{\circ}:=\left\{x^{\prime} \in X^{\prime}:\left|\left\langle y, x^{\prime}\right\rangle\right| \leq\right.$ $1 \forall y \in Y\}$. Observe that the weak topology of $X_{\beta}^{\prime}$ is $\sigma\left(X^{\prime}, X^{\prime \prime}\right)$.

Theorem 9 Let $X$ be a a Grothendieck lcHs which is complete and barrelled and $(T(t))_{t \geq 0} \subset \mathcal{L}(X)$ be a strongly continuous $C_{0}$-semigroup which satisfies (13) and such that $\{C(r)\}_{r \geq 0}$ is equicontinuous. Then the following assertions are equivalent.

(i) $(T(t))_{t \geq 0}$ is mean ergodic. 
(ii) The subspace $Z:=\operatorname{span}\left\{x^{\prime}-T^{\prime}(s) x^{\prime}: s \geq 0, x^{\prime} \in X^{\prime}\right\}$ of $X^{\prime}$ satisfies

$$
\bar{Z}^{\sigma\left(X^{\prime}, X\right)}=\bar{Z}^{\sigma\left(X^{\prime}, X^{\prime \prime}\right)},
$$

that is, the closure of $Z$ in $X_{\sigma^{*}}^{\prime}$ coincides with its closure in $X_{\beta}^{\prime}$.

Proof Observe $\left(T(s)^{\prime}\right)_{s \geq 0} \subseteq \mathcal{L}\left(X_{\beta}^{\prime}\right)$ is a locally equicontinuous $C_{0}$-semigroup (cf. Proposition 6(i)). So, by Remark 1(iii) it is strongly continuous. Also, as $X$ is barrelled, $X_{\beta}^{\prime}$ is quasicomplete, [15, Proposition 11.2.4]. So, the Cesàro means $D(r) x^{\prime}:=\frac{1}{r} \int_{0}^{r} T(s)^{\prime} x^{\prime} d s$, for $x^{\prime} \in X^{\prime}$, exist in $\mathcal{L}\left(X_{\beta}^{\prime}\right)$ for $r>0$ (with $D(0):=I)$. By properties of the Riemann integral it follows that $\left\langle C(r) x, x^{\prime}\right\rangle=$ $\left\langle x, D(r) x^{\prime}\right\rangle$ for $x \in X, x^{\prime} \in X^{\prime}$, i.e., $D(r)=C(r)^{\prime}$ for $r \geq 0$.

(i) $\Rightarrow$ (ii). Since $(T(t))_{t \geq 0}$ is mean ergodic, the operator $P \in \mathcal{L}(X)$ given by $P:=\tau_{s}-\lim _{r \rightarrow \infty} C(r)$ is a projection; see Remark 4(ii). According to (15) we have

$$
\operatorname{Im} P=\operatorname{Fix}(T(\cdot))=\cap_{t \geq 0} \operatorname{Ker}(I-T(t))
$$

and by (16) we have

$$
\text { Ker } P=\overline{\operatorname{span}\{x-T(t) x: t \geq 0, x \in X\}}=\overline{\cup_{t \geq 0} \operatorname{Im}(I-T(t))} .
$$

Moreover, $P^{\prime} \in \mathcal{L}\left(X_{\beta}^{\prime}\right)$ is a projection and so

$$
\operatorname{Im} P^{\prime}=(\operatorname{Ker} P)^{\perp}, \quad \operatorname{Ker} P^{\prime}=(\operatorname{Im} P)^{\perp},
$$

$[20$, p.2, (5)\&(6)]. It then follows from (32) and (34) that

$$
\begin{aligned}
& \text { Ker } P^{\prime}=(\operatorname{Im} P)^{\perp}=\left[\cap_{t \geq 0} \operatorname{Ker}(I-T(t))\right]^{\perp} \\
& =\overline{\operatorname{span}\left(\cup_{s \geq 0} \operatorname{Im}\left(I-T(s)^{\prime}\right)\right)^{\sigma\left(X^{\prime}, X\right)}} \\
& ={\overline{\operatorname{span}\left\{x^{\prime}-T(s)^{\prime} x^{\prime}: s \geq 0, x^{\prime} \in X^{\prime}\right\}}}^{\sigma\left(X^{\prime}, X\right)}=\bar{Z}^{\sigma\left(X^{\prime}, X\right)} \text {, }
\end{aligned}
$$

where we also used $\left[\cap_{t \geq 0} \operatorname{Ker}(I-T(t))\right]^{\perp}={\overline{\operatorname{span}\left(\cup_{s \geq 0} \operatorname{Im}\left(I-T(s)^{\prime}\right)\right)}}^{\sigma\left(X^{\prime}, X\right)}$, $\left[19\right.$, p.247, (11)], and $[\operatorname{Ker}(I-T(s))]^{\perp}={\overline{\operatorname{Im}\left(I-T(s)^{\prime}\right)}}^{\sigma\left(X^{\prime}, X\right)},[20$, p.2, (6)]. It follows that $\bar{Z}^{\sigma\left(X^{\prime}, X^{\prime \prime}\right)} \subseteq \bar{Z}^{\sigma\left(X^{\prime}, X\right)} \subseteq \operatorname{Ker} P^{\prime}$.

Conversely, fix $x^{\prime} \in \operatorname{Ker} P^{\prime}$. Then, for $x \in X$, we have $\left\langle x, D(r) x^{\prime}\right\rangle=$ $\left\langle C(r) x, x^{\prime}\right\rangle \rightarrow\left\langle P x, x^{\prime}\right\rangle=\left\langle x, P^{\prime} x^{\prime}\right\rangle=0$ as $r \rightarrow \infty$, i.e., $\lim _{r \rightarrow \infty} D(r) x^{\prime}=0$ in $X_{\sigma^{*}}^{\prime}$. As $X$ is a Grothendieck space, it follows $D\left(r_{n}\right) x^{\prime} \rightarrow 0$ in $\left(X^{\prime}, \sigma\left(X^{\prime}, X^{\prime \prime}\right)\right)$, whenever $r_{n} \rightarrow \infty$ in $(0, \infty)$. Moreover, the Riemann integral

$$
x^{\prime}-D\left(r_{n}\right) x^{\prime}=\frac{1}{r_{n}} \int_{0}^{r_{n}}\left(x^{\prime}-T(s)^{\prime} x^{\prime}\right) d s,
$$

exists via continuity of the $X_{\beta}^{\prime}$-valued function $s \mapsto\left(I-T(s)^{\prime}\right) x^{\prime}$. Applying Lemma 1(ii) to $\left(T(s)^{\prime}\right)_{s \geq 0} \subseteq \mathcal{L}\left(X_{\beta}^{\prime}\right)$ we have $\left(x^{\prime}-D\left(r_{n}\right) x^{\prime}\right) \in \bar{Z}^{X_{\beta}^{\prime}}=$ $\bar{Z}^{\sigma\left(X^{\prime}, X^{\prime \prime}\right)}$. Letting $n \rightarrow \infty$ in (36) we deduce that $x^{\prime} \in \bar{Z}^{\sigma\left(X^{\prime}, X^{\prime \prime}\right)}$. This shows Ker $P^{\prime} \subseteq \bar{Z}^{\sigma\left(X^{\prime}, X^{\prime \prime}\right)}$ and hence, establishes equality in (31). 
(ii) $\Rightarrow\left(\right.$ i). Suppose (31) holds. As $\operatorname{Fix}(T(\cdot))=\cap_{t \geq 0} \operatorname{Ker}(I-T(t))$, the last three equalities in (35) (which do not require $(T(t))_{t \geq 0}$ to be mean ergodic) imply that $[\operatorname{Fix}(T(\cdot))]^{\perp}=\bar{Z}^{\sigma\left(X^{\prime}, X^{\prime \prime}\right)}$. Also, it follows from [15, Proposition $8.2 .1(\mathrm{f})]$ that

$$
\left[\overline{\operatorname{span} \cup_{t \geq 0} \operatorname{Im}(I-T(t))}\right]^{\perp}=\cap_{s \geq 0} \operatorname{Ker}\left(I-T(s)^{\prime}\right)=\operatorname{Fix}\left(T(\cdot)^{\prime}\right) .
$$

Combining this with $(Y \oplus W)^{\perp}=Y^{\perp} \cap W^{\perp}$ for closed subspaces $Y, W \subseteq X$ and observing that $\operatorname{Fix}(T(\cdot)) \cap \overline{\operatorname{span}\{x-T(t) x: t \geq 0, x \in X\}}=\{0\}$ (cf. Lemma $3(\mathrm{ii})$ ) we have (cf. also (37)) that

$$
\begin{aligned}
{[\operatorname{Fix}(T(\cdot))} & \oplus \overline{\operatorname{span}\{x-T(t) x: t \geq 0, x \in X\}}]^{\perp} \\
& =[\operatorname{Fix}(T(\cdot))]^{\perp} \cap[\overline{\operatorname{span}\{x-T(t) x: t \geq 0, x \in X\}}]^{\perp} \\
& =\bar{Z}^{\sigma\left(X^{\prime}, X^{\prime \prime}\right)} \cap \operatorname{Fix}\left(T(\cdot)^{\prime}\right) .
\end{aligned}
$$

But, equicontinuity of $\{C(r)\}_{r \geq 0}$ implies equicontinuity of $\left\{D(r)=C(r)^{\prime}\right\}_{r \geq 0} \subseteq$ $\mathcal{L}\left(X_{\beta}^{\prime}\right)$ (by a similar argument for local equicontinuity as in the proof of Proposition 6(i)). Hence, by the proof of Lemma 3(ii) applied to $\left(T(s)^{\prime}\right)_{s \geq 0}$ in $X_{\beta}^{\prime}$ we see that $\bar{Z}^{\sigma\left(X^{\prime}, X^{\prime \prime}\right)} \cap \operatorname{Fix}\left(T(\cdot)^{\prime}\right)=\bar{Z}^{X_{\beta}^{\prime}} \cap \operatorname{Fix}\left(T(\cdot)^{\prime}\right)=\{0\}$. It follows that the space $\operatorname{Fix}(T(\cdot)) \oplus \overline{\operatorname{span}\{x-T(t) x: t \geq 0, x \in X\}}$ is dense in $X$. Then Lemma 3 (ii) yields

$$
X=\operatorname{Fix}(T(\cdot)) \oplus \overline{\operatorname{span}\{x-T(t) x: t \geq 0, x \in X\}} .
$$

To see $(T(t))_{t \geq 0}$ is mean ergodic, for $x \in X$ write $x=y+z$ with $y \in$ $\operatorname{Fix}(T(\cdot))$ and $z \in \overline{\operatorname{span}\{x-T(t) x: t \geq 0, x \in X\}}$. By Remark $5(\mathrm{v})$ we have $\lim _{r \rightarrow \infty} C(r) z=0$. Also, $C(r) y=\frac{1}{r} \int_{0}^{r} T(s) y d s=y$ for $r>0$. So, $\lim _{r \rightarrow \infty} C(r) x=$ $y$ exists in $X$.

We end this section with an application of the previous result.

Corollary 3 Let $X$ be a Grothendieck lcHs which is complete and barrelled and $(T(t))_{t \geq 0} \subseteq \mathcal{L}(X)$ be a mean ergodic, strongly continuous $C_{0}$-semigroup which satisfies $\lim _{t \rightarrow \infty} \frac{T(t)}{t}=0$ in $\mathcal{L}_{b}(X)$. Then $\left(T(s)^{\prime}\right)_{s \geq 0} \subseteq \mathcal{L}\left(X_{\beta}^{\prime}\right)$ is mean ergodic.

Proof By Remark 1(i), $(T(t))_{t \geq 0}$ is locally equicontinuous. The hypotheses on $(T(t))_{t \geq 0}$ imply $(13)$. As $r \mapsto C(r)$ is continuous from $[0, \infty)$ into $\mathcal{L}_{s}(X)$ (see Lemma $1(\mathrm{vi}))$ and $P:=\tau_{s}-\lim _{r \rightarrow \infty} C(r)$ exists, $\{C(r)\}_{r \geq 0}$ is bounded in $\mathcal{L}_{s}(X)$ and so, by barrelledness of $X$, is equicontinuous. By Remark 4(ii), $P \in \mathcal{L}(X)$ is a projection.

Lemma 3(i) gives $\operatorname{Im} P^{\prime}=\operatorname{Fix}\left(T(\cdot)^{\prime}\right)$. In the proof of (i) $\Rightarrow($ ii) in Theorem 9 it was shown (with the notation from there) that $\operatorname{Ker} P^{\prime}=\bar{Z}^{\sigma\left(X^{\prime}, X\right)}$; see (35). Then Theorem 9 itself gives $\operatorname{Ker} P^{\prime}=\bar{Z}^{\sigma\left(X^{\prime}, X^{\prime \prime}\right)}=\bar{Z}^{X_{\beta}^{\prime}}$. So, $X_{\beta}^{\prime}=$ $\operatorname{Im} P^{\prime} \oplus \operatorname{Ker} P^{\prime}$ yields $X_{\beta}^{\prime}=\operatorname{Fix}\left(T(\cdot)^{\prime}\right) \oplus \bar{Z}^{X_{\beta}^{\prime}}$. Given $x^{\prime} \in X^{\prime}$, write $x^{\prime}=$ $u^{\prime}+v^{\prime}$ with $u^{\prime} \in \operatorname{Fix}\left(T(\cdot)^{\prime}\right)$ and $v^{\prime} \in \bar{Z}^{X_{\beta}^{\prime}}$. It was shown in the proof of 
Lemma 3(i) that $C(r)^{\prime} u^{\prime}=u^{\prime}$ for $r>0$. Moreover, $\left(T(s)^{\prime}\right)_{s \geq 0} \subseteq \mathcal{L}\left(X_{\beta}^{\prime}\right)$ is a locally equicontinuous $C_{0}$-semigroup (cf. Proposition 6(i))). Then the Cesàro means $D(r) x^{\prime}:=\frac{1}{r} \int_{0}^{r} T(s)^{\prime} x^{\prime} d s$, for $x^{\prime} \in X^{\prime}$, of $\left(T(s)^{\prime}\right)_{s \geq 0}$ exist (as Riemann integrals) in $\mathcal{L}_{s}\left(X_{\beta}^{\prime}\right)$ with $D(r)=C(r)^{\prime}, r>0$; see the proof of Theorem 9. Then $\lim _{r \rightarrow \infty} D(r) u^{\prime}=u^{\prime}$ in $X_{\beta}^{\prime}$.

To show $\left(T(s)^{\prime}\right)_{s \geq 0}$ is mean ergodic, it remains to verify $\lim _{r \rightarrow \infty} D(r) v^{\prime}=$ 0 in $X_{\beta}^{\prime}$, for $v^{\prime} \in \bar{Z}^{X_{\beta}^{\prime}}$. This follows from Remark $5(\mathrm{v})$ applied to $\left(T(s)^{\prime}\right)_{s \geq 0} \subseteq$ $\mathcal{L}\left(X_{\beta}^{\prime}\right)$, provided we check the hypotheses hold. As noted before, $X_{\beta}^{\prime}$ is quasicomplete and it was just observed above that $\left(T(s)^{\prime}\right)_{s \geq 0}$ is a locally equicontinuous $C_{0}$-semigroup on $X_{\beta}^{\prime}$. As $(T(t))_{t \geq 0} \subseteq \mathcal{L}(X)$ is mean ergodic and $X$ is barrelled, Remark 5(ii) implies $\{C(r)\}_{r \geq 0} \subseteq \mathcal{L}(X)$ is equicontinuous. This implies equicontinuity of $\{D(r)\}_{r \geq 0} \subseteq \mathcal{L}\left(X_{\beta}^{\prime}\right)$; cf. proof of (ii) $\Rightarrow$ (i) in Theorem 9. It remains to show that $\frac{T(r)^{\prime}}{r} \rightarrow 0$ in $\mathcal{L}_{s}\left(X_{\beta}^{\prime}\right)$ as $r \rightarrow \infty$.

To this effect, fix $x^{\prime} \in X^{\prime}$. Then there exist $q \in \Gamma_{X}$ and $C>0$ such that $\left|\left\langle x, x^{\prime}\right\rangle\right| \leq C q(x)$ for each $x \in X$. Now, for $B \in \mathcal{B}(X)$, we have

$p^{B}\left(\frac{T(r)^{\prime} x^{\prime}}{r}\right)=\sup _{x \in B}\left|\left\langle\frac{T(r) x}{r}, x^{\prime}\right\rangle\right| \leq C \sup _{x \in B} q\left(\frac{T(r)}{r}\right)=C q_{B}\left(\frac{T(r)}{r}\right), \quad r>0$,

with $q_{B}$ the continuous seminorm in $\mathcal{L}_{b}(X)$ corresponding to $q \in \Gamma_{X}, B \in$ $\mathcal{B}(X)$. As $\tau_{b}-\lim _{r \rightarrow \infty} \frac{T(r)}{r}=0$ (by hypothesis), it follows $p^{B}\left(\frac{T(r)^{\prime} x^{\prime}}{r}\right) \rightarrow 0$. But, this is for arbitrary $x^{\prime} \in X^{\prime}, B \in \mathcal{B}(X)$, i.e., $\frac{T(r)^{\prime}}{r} \rightarrow 0$ in $\mathcal{L}_{s}\left(X_{\beta}^{\prime}\right)$ as $r \rightarrow \infty$.

\section{Appendix: vector-valued Riemann integrals.}

A partition $P$ of a compact interval $[a, b] \subseteq \mathbb{R}$ is any finite set of numbers $a=t_{0}<t_{1}<\ldots<t_{n}=b$, for some $n \in \mathbb{N}$. We write $P=\left\{t_{k}\right\}_{k=0}^{n}$ and set $|P|:=\max \left\{\left(t_{k}-t_{k-1}\right): 1 \leq k \leq n\right\}$. Define $[P]:=\prod_{k=1}^{n}\left[t_{k-1}, t_{k}\right] \subseteq \mathbb{R}^{n}$. The set of all partitions of $[a, b]$ is denoted by $\mathcal{P}([a, b])$. Given $P, Q \in \mathcal{P}([a, b])$ and elements $\xi \in[P], \eta \in[Q]$ define $(P, \xi) \geq(Q, \eta)$ to mean $|P| \leq|Q|$. Then $\geq$ turns $\mathcal{D}:=\cup_{P \in \mathcal{P}([a, b])}\{(P, \xi): \xi \in[P]\}$ into a directed set.

Let $X$ be a lcHs and $f:[a, b] \rightarrow X$ be a bounded function. Given $P=$ $\left\{t_{k}\right\}_{k=0}^{n} \in \mathcal{P}([a, b])$ and $\xi=\left(\xi_{1}, \ldots, \xi_{n}\right) \in[P]$, the element

$$
R(f, P, \xi)=\sum_{k=1}^{n} f\left(\xi_{k}\right)\left(t_{k}-t_{k-1}\right) \in X
$$

is called the Riemann sum of $f$ relative to $P$ and $\xi$. Then $\{R(f, P, \xi)$ : $(P, \xi) \in \mathcal{D}\} \subseteq X$ is a net in $X$. Recall that $f$ is Riemann integrable if the net $\{R(f, P, \xi):(P, \xi) \in \mathcal{D}\}$ converges to an element of $X$, denoted by $\int_{a}^{b} f(t) d t$. More precisely, for every $\varepsilon>0$ and $p \in \Gamma_{X}$ there is $(\bar{P}, \bar{\xi}) \in \mathcal{D}$ such that $p\left(R(f, P, \xi)-\int_{a}^{b} f(t) d t\right)<\varepsilon$, for all $(P, \xi) \geq(\bar{P}, \bar{\xi})$. Equivalently, there is $\delta>0$ (i.e., $\delta:=|\bar{P}|)$ such that $\sup _{\xi \in[P]} p\left(R(f, P, \xi)-\int_{a}^{b} f(t) d t\right)<\varepsilon$, for all $P \in \mathcal{P}([a, b])$ with $|P|<\delta$. 
Theorem 10 Let $X$ be a sequentially complete lcHs and $f:[a, b] \rightarrow X$ be a continuous function. Then $f$ is Riemann integrable.

Proof It suffices to consider $[a, b]=[0,1]$. Fix $p \in \Gamma_{X}$ and $\varepsilon>0$. The space $X$ equipped with the pseudometric $(x, y) \mapsto p(x-y)$ is a uniform space. Since $[0,1]$ is a compact uniform space, $f$ is uniformly continuous, $[16$, Ch. 6 , Theorem 31]. So, there is $\delta>0$ such that $p(f(s)-f(t))<\varepsilon$ whenever $s, t \in[0,1]$ satisfy $|s-t|<\delta$. Fix any $\bar{P} \in \mathcal{P}([0,1])$ with $|\bar{P}|<\delta$. Suppose that $P, Q \in \mathcal{P}([0,1])$ satisfy $P \geq \bar{P}$ and $Q \geq \bar{P}$, and consider the common refinement $P \cup Q$. Let $\xi \in[P]$ and $\xi^{\prime} \in[P \cup Q]$.

Let $[u, v]$ be any subinterval of $[0,1]$ determined by two adjacent points of $P$ and let $r$ be that coordinate of $\xi$ such that $r \in[u, v]$. Note that $|v-u|<\delta$ and $\left|r-t_{k}\right|<\delta$ for every $k=0, \ldots, n$, where $\left\{t_{k}\right\}_{k=0}^{n}$ are those points of $P \cup Q$ which lie in $[u, v]$ and hence, form a partition of $[u, v]$. Then

$$
f(r)(v-u)-\sum_{k=1}^{n} f\left(\xi_{k}^{\prime}\right)\left(t_{k}-t_{k-1}\right)=\sum_{k=1}^{n}\left(f(r)-f\left(\xi_{k}^{\prime}\right)\right)\left(t_{k}-t_{k-1}\right)
$$

and hence,

$$
p\left(f(r)(v-u)-\sum_{k=1}^{n} f\left(\xi_{k}^{\prime}\right)\left(t_{k}-t_{k-1}\right)\right) \leq \varepsilon \sum_{k=1}^{n}\left(t_{k}-t_{k-1}\right)=\varepsilon(v-u) .
$$

It follows that $p\left(R(f, P, \xi)-R\left(f, P \cup Q, \xi^{\prime}\right)\right)<\varepsilon$, for all choices of $\xi \in[P]$ and $\xi^{\prime} \in[P \cup Q]$. A similar argument yields $p\left(R(f, Q, \eta)-R\left(f, P \cup Q, \xi^{\prime}\right)\right)<\varepsilon$, for all choices of $\eta \in[Q]$ and $\xi^{\prime} \in[P \cup Q]$. Combining the previous two inequalities yields $p(R(f, P, \xi)-R(f, Q, \eta)<2 \varepsilon$, for all $\xi \in[P], \eta \in[Q]$, whenever $P, Q \in \mathcal{P}([0,1])$ satisfy $P \geq \bar{P}$ and $Q \geq \bar{P}$. This establishes that the Riemann sums form a Cauchy net in $X$.

The partitions $\left(P_{n}, \xi_{[n]}\right)$ with $P_{n}:=\left\{\frac{k}{n}: k=0, \ldots, n\right\}$ and $\xi_{[n]}:=$ $\left(0, \frac{1}{n}, \frac{2}{n}, \ldots, 1\right) \in\left[P_{n}\right]$, for $n \in \mathbb{N}$, form a countable cofinal subset of $\mathcal{D}$. Since $\left(P_{n}, \xi_{[n]}\right) \leq\left(P_{m}, \xi_{[m]}\right)$ whenever $n \leq m$, this ensures that the Riemann sums

$$
R\left(f, P_{n}, \xi_{[n]}\right)=\frac{1}{n} \sum_{k=1}^{n} f\left(\frac{k}{n}\right), \quad n \in \mathbb{N}
$$

form a Cauchy sequence in $X$ which then converges to some $x \in X$. To see that the net of all Riemann sums of $f$ also converges to $x$, fix $\varepsilon>0$ and $p \in \Gamma_{X}$. Then there is $\bar{P} \in \mathcal{P}([0,1])$ such that $p(R(f, P, \xi)-R(f, Q, \eta))<\varepsilon$, for all $\xi \in[P], \eta \in[Q]$, whenever $P, Q \in \mathcal{P}([0,1])$ satisfy $P, Q \geq \bar{P}$. Choose $N \in \mathbb{N}$ such that $(\bar{P}, \xi) \leq\left(P_{n}, \xi_{[n]}\right)$ for all $n \geq N$ and $\xi \in[\bar{P}]$. Via the previous inequality we have

$$
p\left(R(f, P, \xi)-R\left(f, P_{n}, \xi_{[n]}\right)\right)<\varepsilon, \quad \forall n \geq N, \xi \in[P],
$$

whenever $P \in \mathcal{P}([0,1])$ satisfies $P \geq \bar{P}$ (i.e. $|P| \leq|\bar{P}|$ ). Let $n \rightarrow \infty$ in (39) gives $p(R(f, P, \xi)-x) \leq \varepsilon$, for all $P \geq \bar{P}, \xi \in[P]$. Hence, the net of Riemann sums of $f$ converges to $x$. 
A function $f: J \rightarrow X$, with $J \subseteq \mathbb{R}$ an open interval, is differentiable at $t_{0} \in J$ if there is $f^{\prime}\left(t_{0}\right) \in X$ satisfying $\lim _{t \rightarrow t_{0}} \frac{f(t)-f\left(t_{0}\right)}{t-t_{0}}=f^{\prime}\left(t_{0}\right)$ with the limit existing in $X$. We write $f \in C^{1}(J, X)$ if $f$ is diffentiable at each point of $J$ and $f^{\prime}: J \rightarrow X$ is continuous. A function $f:[a, b] \rightarrow X$ is a $C^{1}$-function means there is an open interval $J \supseteq[a, b]$ and $g \in C^{1}(J, X)$ such that the restriction $\left.g\right|_{[a, b]}=f$.

Since the proofs of the following properties follow via routine duality arguments, approximation via Riemann sums, and the scalar theory of Riemann integration, we omit the details.

Proposition 11 Let $X$ be a sequentially complete lcHs, the real numbers a, $b$ satisfy $a<b$ and $f:[a, b] \rightarrow X$ be a continuous function.

(i) $\left\langle\int_{a}^{b} f(t) d t, x^{\prime}\right\rangle=\int_{a}^{b}\left\langle f(t), x^{\prime}\right\rangle d t$ for all $x^{\prime} \in X^{\prime}$.

(ii) $\int_{a}^{u} f(t) d t+\int_{u}^{b} f(t) d t=\int_{a}^{b} f(t) d t$ for all $u \in \mathbb{R}$ with $a<u<b$.

(iii) $\int_{a}^{b}(f \circ g)(t) g^{\prime}(t) d t=\int_{g(a)}^{g(b)} f(t) d t$ for all $g \in C^{1}(\mathbb{R}, \mathbb{R})$.

(iv) $\int_{a}^{b} f(t) d t$ lies in the closed convex hull of the compact set $(b-a) f([a, b])$.

(v) If $f:[a, b] \rightarrow X$ is a $C^{1}$-function, then $f(b)-f(a)=\int_{a}^{b} f^{\prime}(t) d t$.

(vi) If $T \in \mathcal{L}(X)$, then $T\left(\int_{a}^{b} f(t) d t\right)=\int_{a}^{b}(T \circ f)(t) d t$.

(vii) $p\left(\int_{a}^{b} f(t) d t\right) \leq \int_{a}^{b} p(f(t)) d t$ for all $p \in \Gamma_{X}$.

\section{References}

1. A.A. Albanese, J. Bonet, W.J. Ricker, Mean ergodic operators in Fréchet spaces, Ann. Acad. Sci. Fenn. Math. 34, 401-436 (2009).

2. A.A. Albanese, J. Bonet, W.J. Ricker, Grothendieck spaces with the Dunford-Pettis property, Positivity, 14, 145-164 (2010).

3. A.A. Albanese, J. Bonet, W.J. Ricker, On mean ergodic operators, In: Vector Measures, Integration and Related Topics, G.P. Curbera et. al. (Eds), Operator Theory: Advances and Applications 201, Birkhäuser Verlag Basel, pp. 1-20 (2010).

4. A.A. Albanese, J. Bonet, W.J. Ricker, $C_{0}$-semigroups and mean ergodic operators in a class of Fréchet spaces, J. Math. Anal. Appl. 365, 142-157 (2010).

5. J. Bonet, W.J. Ricker, Schauder decompositions and the Grothendieck and the DunfordPettis properties in Köthe echelon spaces of infinite order, Positivity 11, 77-93 (2007).

6. J. Bonet, W.J. Ricker, Mean ergodicity of multiplication operators in weighted spaces of holomorphic functions, Arch. Math. 92, 428-437 (2009).

7. J. Bonet, B. de Pagter, W.J. Ricker, Mean ergodic operators and reflexive Fréchet lattices, Proc. Roy. Soc. Edinburgh Sect. A, to appear.

8. N. Dunford, J.T. Schwartz, Linear Operators I: General Theory (2nd printing), WileyInterscience, New York (1964).

9. W.F. Eberlein, Abstract ergodic theorems and weak almost periodic functions, Trans. Amer. Math. Soc. 67, 217-240 (1949).

10. R.E. Edwards, Functional Analysis, Reinhart and Winston, New York (1965).

11. K.-J. Engel, R. Nagel, One-Parameter Semigroups for Linear Evolution Equations, Springer, New-York (1999).

12. K. Floret, Weakly Compact Sets, LNM 801, Springer, Berlin-Heidelberg (1980).

13. V.P. Fonf, M. Lin, P. Wojtaszczyk, Ergodic characterizations of reflexivity in Banach spaces, J. Funct. Anal. 187, 146-162 (2001). 
14. E. Hille, R.S. Phillips, Functional Analysis and Semigroups, 4th printing Rev. Ed., American Math. Soc., Providence (1981).

15. H. Jarchow, Locally Convex Spaces, B.G. Teubner, Stuttgart (1981).

16. J.L. Kelley, General Topology, Rev. Ed., D. van Nostrand Co., Princeton-New York (1961).

17. H. Komatsu, Semi-groups of operators in locally convex spaces, J. Math. Soc. Japan 16, 230-262 (1964)

18. T. Komura, Semigroups of operators in locally convex spaces, J. Funct. Anal. 2, 258-296 (1968).

19. G. Köthe, Topological Vector Spaces I, 2nd Rev. Ed., Springer Verlag, Berlin-HeidelbergNew York (1983).

20. G. Köthe, Topological Vector Spaces II, Springer Verlag, Berlin-Heidelberg-New York (1979).

21. U. Krengel, Ergodic Theorems, Walter de Gruyter, Berlin (1985).

22. H.P. Lotz, Tauberian theorems for operators on $L^{\infty}$ and similar spaces, In: "Functional Analyis: Surveys and Recent Results III", K.D. Bierstedt and B. Fuchssteiner (Eds.), North Holland, Amsterdam, pp. 117-133 (1984).

23. H.P. Lotz, Uniform convergence of operators on $L^{\infty}$ and similar spaces, Math. Z. 190, 207-220 (1985)

24. R. Meise, D. Vogt, Introduction to Functional Analysis, Clarendon Press, Oxford (1997).

25. I. Miyadera, Semigroups of operators in Fréchet spaces and applications to partial differential operators, Tôhoku Math. J. 11, 162-183 (1959).

26. D. Mugnolo, A semigroup analogue of the Fonf-Lin-Wojtaszczyk characterization of reflexive Banach spaces with a basis, Studia Math. 164, 243-251 (2004).

27. K. Piszczek, Quasi-reflexive Fréchet spaces and mean ergodicity, J. Math. Anal. Appl. 361, 224-233 (2010)

28. K. Piszczek, Barrelled spaces and mean ergodicity, RACSAM 104, 5-11 (2010).

29. W. Rudin, Functional Analysis, McGraw-Hill, New York (1973).

30. R. Sato, On a mean ergodic theorem, Proc. Amer. Math. Soc. 83, 563-564 (1981).

31. H.H. Schaefer, Banach Lattices and Positive Operators, Springer, Berlin-Heidelberg (1974).

32. S.-Y. Shaw, Ergodic projections of continuous and discrete semigroups, Proc. Amer. Math. Soc. 78, 69-76 (1980).

33. K. Yosida, Functional Analysis, Springer-Verlag, Berlin, 1980. 